\title{
PARTIALLY ACYCLIC MANIFOLD DECOMPOSITIONS YIELDING GENERALIZED MANIFOLDS
}

\author{
DAVID F. SNYDER
}

\begin{abstract}
Let $G$ be an upper semicontinuous decomposition (uscd) of the $(n+k)$-manifold $M$ into subcontinua having the shape of closed orientable $n$ manifolds $(2<n, k)$. We define $G$ to be $j$-acyclic if for every element $g$ of $G$ the reduced Čech homology of $g$ vanishes up through dimension $j$. The primary objective of this investigation is to determine the local connectivity properties of the decomposition space $B=M / G$ if $G$ is $(k-2)$-acyclic and $B$ is finite dimensional. The Leray-Grothendieck spectral sequence of the decomposition map $p$ is analyzed, which relegates the principal part of the investigation to studying the structure of the Leray sheaf of $p$ and its relation to the local cohomology of $B$. Let $E$ denote the subset of $B$ over which the Leray sheaf is not locally constant, $K$ the subset of $E$ over which the Leray sheaf is not locally Hausdorff, and $D=E-K$. Then we get as our main result, which extends work of R. J. Daverman and J. J. Walsh, and generalizes a result of D. S. Coram and P. Duvall as well,
\end{abstract}

Theorem. Let $G$ be a $(k-2)$-acyclic decomposition of the $(n+k)$-manifold $M$ such that $k<n+2, B=M / G$ is finite dimensional, and the set $E$ does not locally separate $B$. Then $B$ is a generalized $k$-manifold, if either $k=n+1$, or $k<n+1$ and $M$ is orientable.

\section{INTRODUCTION}

We mainly consider here decompositions whose elements are closed orientable $n$-manifolds with

$$
\widetilde{H}_{i}(g)=0, \quad i \in\{0,1, \ldots, k-2\},
$$

where $2<k<n+2$, extending Theorem 3.1 in [DW4], as well as weakly extending the main result in [CD2].

An upper semicontinuous decomposition (uscd) $G$ of a metric space $X$ is a collection of pairwise disjoint compact subsets which cover $X$ so that for each neighborhood $U$ of an element $g \in G$ the set $\bigcup\left\{g^{\prime} \mid g^{\prime} \in G, g^{\prime} \subset U\right\}$ (called

Received by the editors March 31, 1989.

1980 Mathematics Subject Classification (1985 Revision). Primary 57N15; Secondary 55M25, 55N30, 55T99, 57P99.

Key words and phrases. Upper semicontinuous decomposition, Leray sheaf, spectral sequence, homology sphere.

I sincerely thank my advisor, R. J. Daverman, for his patient encouragement and John Bryant for his suggestions. 
the saturate of $U$ by $G$ ) is an open subset of $X$. The decomposition space, denoted $X / G$, is the quotient space of $X$ under the relation $\sim$, where $x_{1} \sim x_{2}$ if, and only if, there is $g \in G$ with $x_{1}, x_{2} \in g$. The quotient map is called the decomposition map. This map is closed.

As usual, $\mathbf{Z}$ denotes the integers and $\mathbf{Z}_{p}$ denotes the integers modulo $p$. For the functors of Algebraic Topology we use the notation of $[\mathrm{S}, \mathrm{Br} 1]$, where the coefficient module is taken to be $\mathbf{Z}$ unless indicated otherwise.

A codimension- $k$ manifold decomposition will signify a uscd of an $(n+$ $k$ )-manifold $M$ into subcontinua having the shape of closed orientable $n$ manifolds. If for $i<n$ we have $\widetilde{H}_{j}(g)=0$ for all $j \leq i$ we call $G$ an $i$-acyclic manifold decomposition. Note that a codimension- $n$ manifold decomposition of an $n$-manifold is cell-like. The codimension is emphasized here since it is a primary limiting factor in analyzing the decomposition space.

To put the results and techniques of this paper into proper context, the literature pertaining to codimension- 2 manifold decompositions, then higher codimensional decompositions, is reviewed. The reader is also pointed to the survey article by R. J. Daverman [D4] as an excellent information source.

In the codimension- 2 case, it is important to point out certain classical results of the 1930's which use very specific circle decompositions of 3-manifolds to display the topological structure of either the decomposition space or the source (i.e., decomposed) space. In [Ho], H. Hopf presented certain maps from the 3-sphere to the 2-sphere, including the famous Hopf fibration. This latter map represents a generator of $\Pi_{3}\left(S^{2}\right)$. Soon after, H. Seifert [Se] developed his elegant theory of Seifert fibered spaces. Each of these fiberings is a uscd of a 3-manifold into circles, though for a decomposition theorist these are atypically rigid decompositions. However, because of this rigidity, these spaces are useful aids in the analysis of geometric structures on 2-manifolds (see [Sc, BoSb]), and have interesting geometric structures themselves as well [Th].

In the 1970's D. S. Coram and P. F. Duvall investigated decompositions of the 3-sphere into compacta having the shape of the circle; for example, they prove [CD3] that the decomposition map is approximable by either the Hopf fibration or a Seifert fiber map (R. J. Daverman and J. J. Walsh proved the decomposition space to be the 2-sphere [DW2]). The techniques that Coram and Duvall applied are fundamental for following results in this area.

In particular, winding functions are primary tools of that investigation. Suppose $G$ to be a uscd of a manifold $M$ into closed orientable submanifolds of dimension $n$. Pick an element $g$ of $G$. Since $g$ is an ANR there is a neighborhood $U$ of $g$ in $M$ which retracts by a map $r$ to $g$.

For a given element $g^{\prime} \subset U$ of $G$ we define the $n$-winding of $g^{\prime}$ about $g$ by $\left|\left(r \mid g^{\prime}\right)^{*}(1)\right|$, where $\left(r \mid g^{\prime}\right)^{*}: H^{n}(g) \rightarrow H^{n}\left(g^{\prime}\right)$ is the induced map on cohomology $\left(H^{n}\left(g^{\prime}\right)=H^{n}(g)=\mathbf{Z}\right)$. Let $b, b^{\prime}$, and $W$ denote the images under the decomposition map of $g, g^{\prime}$, and the saturate of $U$ by $G$, respectively. Note that $W$ is open in $M / G$. We define an $n$-winding function at $b$ on $W$, 
denoted $\alpha_{b}$; its value at $b^{\prime}$ is the $n$-winding of $g^{\prime}$ about $g$. This function is lower semicontinuous on $W$. A Baire category argument can then be used to show that it is continuous on a dense open subset of $W$. Also, $\alpha_{x_{0}}\left(x_{2}\right)=$ $-\alpha_{x_{0}}\left(x_{1}\right) \cdot \alpha_{x_{1}}\left(x_{2}\right)$ whenever these quantities are defined.

Using winding functions, amongst other things, Daverman and Walsh investigated more general problems than those considered by Coram and Duvall. First they broadened the scope of the problem to include codimension- 2 decompositions of an $(n+2)$-manifold into spheres [DW2]. Further extending the scope of their investigation to any codimension-two decomposition of an orientable manifold into closed orientable submanifolds, they prove (in [DW3]), for example, that the decomposition space is a 2-manifold. The decomposition map is shown to be an approximate fibration if a locally finite set of points is removed from the resulting 2-manifold. Daverman extended the result to include the case where $G$ consists of nonorientable closed $n$-manifolds.

One would expect that manifold decompositions of codimension higher than 2 are not going to behave as nicely as those of codimension- 2 and this is the case. Daverman and Walsh give an example of a 5-manifold decomposed into orientable surfaces so that the decomposition space is not a generalized manifold [DW4]. One of the elements is a torus, the others are spheres. So some restriction on the homology of the elements is usually dictated if one expects the decomposition space to be a generalized manifold.

Yet some theorems may be obtained without restricting the decomposition elements in such a way. Daverman established that codimension-3 manifold decompositions (under the modest restriction of orientability of the elements and source space) have finite-dimensional decomposition spaces [D2].

Of crucial importance to this paper is the result of J. Dydak and J. Segal [DyS2] that shows the decomposition space of a codimension- $k$ manifold decomposition of a manifold is $\mathrm{LC}^{1}$ (this is discussed in more detail in $\S 1.5$ ). In conjunction with Dydak's extension of the Hurewicz Theorem [Hr], this gives a simple way of determining if the decomposition space is an ANR; the problem is reduced to determining the local cohomological connectedness of the space.

Daverman and L. S. Husch establish the strong result that there is an open dense subset of the decomposition space over which the elements are all shape equivalent $[\mathrm{DH}]$.

In [CD2, CD4], Coram and Duvall study Lacher's $k$-sphere mappings (i.e., mappings from the $(2 k+1)$-sphere onto a manifold whose point inverses have the shape of the $k$-sphere [L1]), showing them to be approximate fibrations off of a finite subset of the image. Their paper introduces some of the techniques of $\S 2$, in which some of their results are generalized. Part of the strategy is the same: show a certain subset of the image space to be locally finite. However, Coram and Duvall draw conclusions about the map assuming manifold structure of the image, whereas here the structure on the image is established by carefully studying the map and the local structure on a dense open subset of the image. 
Recent work of Daverman and Walsh uses sheaf theory and spectral sequences to determine certain restrictions of the cohomological dimension of the decomposition space.

Theorem (Daverman-Walsh [DW4, Theorem 1.2]). Suppose $M$ is a complete metrizable space and let $f: M \rightarrow B$ be a proper map such that each set $f^{-1}(y)$ has the shape of a closed connected orientable n-manifold. Then

$$
\operatorname{dim}_{\mathrm{Z}} B \leq \operatorname{dim}_{\mathrm{Z}} M-n .
$$

Here $" \operatorname{dim}_{\mathrm{Z}} B$ " denotes the cohomological dimension of $B[\mathrm{~N}, \mathrm{Na}]$. In the same paper, they also establish, using essentially the Vietoris-Begle Mapping Theorem and winding functions, that if the source space is an orientable $(n+k)$-manifold, $n \geq k \geq 3$, decomposed into continua having the shape of orientable closed $n$-manifolds with trivial reduced homology in dimensions less than $k$ such that the decomposition space is finite dimensional, then the decomposition space is a generalized $k$-manifold. This result is extended and generalized herein.

My investigation followed a route suggested by lacunary phenomena of spectral sequences, along with duality properties of the source manifold.

The main results are now stated.

Theorem 1. Let $M^{2 n+1}$ be a manifold, $n>1$, and $G$ a uscd of $M$ into compacta having the shape of homology $n$-spheres such that $\operatorname{dim} M / G<\infty$. Suppose the discontinuity set $E=D \cup K$ (see $\S \S 1.2$ and 1.4) does not locally separate $B$. Then $M / G$ is a generalized $(n+1)$-manifold. Moreover, $E$ is a locally finite subset of $B$.

This theorem generalizes the main theorem in [CD2] about $k$-sphere mappings, where $M$, the decomposition elements, and the decomposition space are assumed to be spheres. Their conclusion that the map is an approximate fibration off of the locally finite subset if the elements have the shape of the $n$-sphere still follows without assuming the image to be a sphere.

Corollary 1. In addition to the hypotheses of Theorem 1, assume all the elements have the shape of the $n$-sphere. Then the decomposition map is an approximate fibration off of the set $E$.

Corollary 2. There can be no uscd of Euclidean $(2 n+1)$-space, $E^{2 n+1}$, into continua having the shape of homology $n$-spheres such that the set $E$ does not locally separate the decomposition space.

Corollary 3. If $G$ is a uscd of $S^{2 n+1}$ into homology n-spheres with exceptional set not locally separating the decomposition space, then the degeneracy set $K$ consists of at most one point.

Example. Given $n>1$, let $\Sigma_{1}, \Sigma_{2}$ be homology $n$-spheres. Let $M=\Sigma_{1} * \Sigma_{2}$ denote the external join [RS, p. 23] of $\Sigma_{1}$ and $\Sigma_{2}$. Then $M$ is a $(2 n+1)$ sphere [E, C]. Let $G_{1}$ denote the decomposition of $M$ which has all elements, 
but one, as those copies of $\Sigma_{2}$ parallel to the obvious end of the join, the remaining one being the end of the join corresponding to $\Sigma_{1}$. Similarly, $G_{2}$ will be the decomposition of $M$ all of whose elements are parallel to the $\Sigma_{1}$ end of the join, but for one corresponding to the $\Sigma_{2}$ end. The space $M / G_{i}$ is homeomorphic to the suspension of $\Sigma_{i}, i=1,2$ (each is a generalized manifold but not a manifold at the suspension points if $\Sigma_{i}$ is not a sphere). The distinguished element of the decomposition $G_{i}$ represents such an element as described in Corollary 3.

Lest the reader think that the nonmanifold points are always so sparse, recall the example of Daverman and Walsh [DW1] of a "ghastly" generalized $n$ manifold $X^{n} \quad(n>2)$ which is not locally Euclidean at any point, yet crossed with the real line is a manifold. Then $M=X^{n} \times \Sigma$, where $\Sigma$ is any homology $(n-1)$-sphere, is a manifold with a natural decomposition into homology $(n-1)$-spheres yielding $X^{n}$ as the decomposition space. The decomposition map is a trivial bundle over $X$. The point is that the structure of the decomposition space can be highly non-Euclidean if the fibers of the map are not nicely embedded with respect to the manifold structure on $M$.

Theorem 2. Let $M^{n+k}$ be an orientable manifold, $n \geq k \geq 3$. Let $G$ be a uscd of $M$ into compacta having the shape of connected, closed, orientable $n$ manifolds with $\check{H}_{i}(g) \cong 0$ for $0<i<k-1$. If $\mathscr{H}^{n}[p]$ (see $§ 1.1$ ) is Hausdorff, $D$ does not locally separate $B$, and $\operatorname{dim} M / G<\infty$, then $M / G$ is a generalized $k$-manifold.

This theorem extends the result of Daverman and Walsh mentioned above; their hypotheses are identical except " $i<k-1$ " is replaced by " $i<k$ ", and here $G$ is assumed to be nondegenerate.

Corollary 4. There cannot be a nondegenerate uscd $G$ of $(n+k)$-dimensional Euclidean space, $E^{n+k}$, into compacta having the shape of $n$-manifolds, each of which is a product of spheres of dimension at least $k-1$, with the exceptional set $E$ not locally separating the decomposition space.

Finally, it is worth remarking that the hypotheses concerning " $E$ not locally separating" are a function of the method used and may well be superfluous.

\section{TOOLS AND METHODS}

This section contains the bulk of observations needed to understand the more arcane aspects of the proofs in $\S \S 2$ and 3.

$\S 1.1$ discusses presheaves, sheaves, and the Leray sheaf of a closed map. $\S 1.2$ deals with the relationship of winding functions to the Leray sheaf, establishing two useful equivalences. Cohomology with coefficients in the Leray sheaf is discussed in $\S 1.3$ and salient points of the theory of sheaf cohomology are stated. In particular, the Leray spectral sequence of a closed map is described. $\S 1.4$ defines the concept of generalized manifold as used in this paper, briefly describing significant properties of generalized manifolds and ANRs established 
in the literature. The last subsection contains various algebraic lemmas that are used in $\S \S 2$ and 3.

1.1. Sheaves and presheaves. This subsection defines sheaf, presheaf, sections, and the Leray sheaf. For a rigorous and more general treatment of sheaves the reader is referred to the books by Swan [Sw] and Bredon $[\mathrm{Br} 1]$.

Definitions. A sheaf on a topological space $X$ is a pair $(\mathscr{S}, \pi)$, where $\mathscr{S}$ is a topological space and $\pi: \mathscr{S} \rightarrow X$ is an open, continuous map satisfying the following properties:

(1) $\mathscr{S}_{x}=\pi^{-1}(x)$, the stalk of $\mathscr{S}$ at $x$, is a $\mathrm{Z}$-module with the discrete topology for all $x \in X$.

(2) For all $s \in \mathscr{S}$ there exists a neighborhood $U$ of $s$ in $\mathscr{S}$ such that $\left.\pi\right|_{U}$ is a homeomorphism onto its image.

(3) Given $x \in X, s, t \in \mathscr{S}_{x}$, and a neighborhood $U$ of $s+t$, there is a neighborhood $V$ of $s$ and neighborhood $W$ of $t$ such that, for all $y \in \pi(U) \cap \pi(V) \cap \pi(W)$, if $s^{\prime} \in V \cap \mathscr{S}_{y}$ and $t^{\prime} \in W \cap \mathscr{S}_{y}$, then $s^{\prime}+t^{\prime} \in U$.

Given $Y \subset X$, a section of $\mathscr{S}$ over $Y$ is a (continuous) map $\sigma: Y \rightarrow \mathscr{S}$ such that $\pi \circ \sigma$ is the identity. Let $\check{H}^{0}(Y ; \mathscr{S})$ denote the set of sections over $Y$; by (2) and (3) this set is a $\mathrm{Z}$-module. The restriction of $\mathscr{S}$ to $Y$, denoted $\left.\mathscr{S}\right|_{Y}$, is $\left(\pi^{-1}(Y),\left.\pi\right|_{\pi^{-1}(Y)}\right)$.

$\mathscr{S}$ is constant if $\mathscr{S}=X \times G \quad(G$ a $\mathrm{Z}$-module $)$ and $\pi$ is projection onto the first factor. $\mathscr{S}$ is locally constant at $x \in X$ if there exists a neighborhood $U \subset X$ of $x$ such that $\left.\mathscr{S}\right|_{U}$ is constant on $U$.

Note that, if $X$ is Hausdorff, a sheaf $\mathscr{S}$ on $X$ is Hausdorff if, and only if, given any stalk $\mathscr{S}_{x}$, any two points in the stalk can be separated by disjoint open neighborhoods in $\mathscr{S}$. Moreover, if two sections of $\mathscr{S}$ agree at a point, then they agree on an open and closed set.

Given a section $\sigma$ over $Y \subset X$ into $\mathscr{S}$, we define the support of $\sigma$ to be the closure in $Y$ of the set

$$
\left\{y \mid \sigma(y) \neq 0 \in \pi^{-1}(y)\right\} .
$$

A family of supports $\phi$ on $X$ denotes a collection of closed subsets of $X$ with the following properties:

(1) A closed subset of an element of $\phi$ is an element of $\phi$.

(2) $\phi$ is closed under finite unions.

We say $\phi$ is paracompactifying if, in addition, it satisfies:

(3) Each element of $\phi$ is paracompact.

(4) Each element of $\phi$ has a closed neighborhood in $\phi$.

We concern ourselves only with three families of supports: the family of closed subsets of $X$, the family of compact subsets of $X$, or the family consisting of a singleton and the null set. Since our space $X$ will always be locally 
compact metric, the first two of these families are paracompactifying. The last one is not.

Many times we must consider sections with restricted support. Let $H_{\phi}^{0}(Y ; \mathscr{S})$ denote the set of sections of $\mathscr{S}$ over $Y$ with support an element of the family of supports $\phi$ on $Y$.

A presheaf on $X$ is a contravariant functor from the category of inclusions of open subsets of $X$ to the category of homomorphisms of Z-modules. A presheaf $S$ on a space $X$ induces a sheaf $\mathscr{S}$ on $X$ (see [Brl, pp. 2-3]) with the following key properties:

(1) $\mathscr{S}_{x}=\lim _{\longrightarrow} S(U)$, for all $x \in X$, where the direct limit is taken over all neighborhoods of $x$ in $X$.

(2) The topology of $\mathscr{S}$ is generated by sets of the form $\left\{\alpha_{x} \mid x \in U\right\}$, where $U$ is any open subset of $X, \alpha \in S(U)$, and $\alpha_{x}$ is the image of $\alpha$ in $\mathscr{S}_{x}$ under the direct limit.

Given a closed, proper map $p: Y \rightarrow X$, the cohomology presheaf on $X$ in dimension $n \geq 0$ induced by $p$ is given by the functor $U \mapsto \breve{H}^{n}\left(p^{-1}(U) ; \mathbf{Z}\right)$. The sheaf induced by this presheaf, denoted $\mathscr{H}^{n}[p]$, is called the Leray sheaf in dimension $n$ induced by $p$. When there is no confusion as to which map $p$ is being discussed, we write briefly $\mathscr{H}^{n}$.

The novitiate may find it informative to draw $\mathscr{H}^{n}$ for the Hopf map and various Seifert fibrations.

1.2. Relations between winding functions and the Leray sheaf. Inspecting definitions suggests some relationships between the winding functions and the Leray sheaf, as in Theorem 1. Having ascertained these, we can have the luxury of using either tool at certain points in the analyses in the following chapters.

In this subsection, we establish equivalences of a given condition on the sheaf with a condition upon the winding function(s) (for example, local constancy at $b$ with continuity of $\alpha_{b}$ at $b$ ). The subsection closes with a useful corollary, which also follows from more general work of J. Dydak and J. J. Walsh [DyW], as well as being a consequence of results in [DH].

In general, the Leray sheaf is not a Hausdorff space. As noted in $\S 1.1$, if two sections into a Hausdorff sheaf agree at a point, then they agree on the component containing that point. So if the base space of a Hausdorff sheaf is locally connected we expect the module of sections to be particularly amenable to analysis. Thus the importance of the following proposition.

Proposition 1.2.1. Let $G$ be a uscd of $M$ into continua having the shape of orientable $n$-manifolds, with $p$ denoting the decomposition map. The sheaf $\mathscr{H}^{n}$ is Hausdorff if, and only if, each $n$-winding function $\alpha_{b}$ is nonzero on some neighborhood of $b$.

Proof. Note that each stalk of $\mathscr{H}^{n}$ is isomorphic to $\mathbf{Z}$; for $b \in B$ and $\zeta \in \mathbf{Z}$ let $\zeta_{b}$ denote the element of the stalk over $b$ corresponding to $\zeta$ under a given isomorphism: $\zeta_{b}$ is well defined modulo the nontrivial automorphism of $\mathbf{Z}$. 
$\Rightarrow$ Suppose that $\mathscr{H}^{n}$ is Hausdorff. Let $U$ be any neighborhood of $b$. Pick a connected neighborhood $V$ of $b$ in $U$ so that $p^{-1}(V)$ shape deforms in $p^{-1}(U)$ to $p^{-1}(\{b\})$. Let $s: V \rightarrow \mathscr{H}^{n}(V)$ be the section over $V$ such that $s(b)=1_{b}$, and $t$ be the trivial section over $V$.

Suppose there exists a sequence of points $\left\{x_{i}\right\}_{i=1}^{\infty}$ converging to $b$ with the property that $\alpha_{b}\left(x_{i}\right)=0$. Thus, $x_{i}$ lies in $V$ for sufficiently large $i$. Hence, $s\left(x_{i}\right)$ is defined for large $i$ and equals $0_{x_{i}}$; but this means $s(x)=t(x)=0_{x}$ for all $x \in V$, since $\mathscr{H}^{n}$ is Hausdorff and $V$ is connected. In particular, $s(b)=t(b)$, a contradiction.

$(\Leftarrow)$ Suppose that $\mathscr{H}^{n}$ is not Hausdorff. Then there are two points in $\mathscr{H}^{n}$ which cannot be separated by disjoint open sets; in particular, since $B$ is Hausdorff, any two such points must lie in the same stalk, say $\mathscr{H}_{b}^{n}$. Let $\gamma_{b}, \beta_{b} \in \mathscr{H}_{b}^{n}$ be two such points. Find a connected neighborhood $U$ of $b$ in $B$ so that $U$ is contained in the domain of $\alpha_{b}$ and such that there are sections $s_{\gamma}, s_{\beta}: U \rightarrow \mathscr{H}^{n}$ with $s_{\gamma}(b)=\gamma_{b}, s_{\beta}(b)=\beta_{b}$. Note that $U_{\gamma}=s_{\gamma}(U)$ and $U_{\beta}=s_{\beta}(U)$ intersect over arbitrarily small neighborhoods of $b$. Let $s_{\delta} \equiv s_{\gamma}-s_{\beta}, \delta_{b}=\gamma_{b}-\beta_{b}$, and let $s_{0}$ be the restriction of the zero section to $U$. Now the previous remark gives that $s_{\delta}$ and $s_{0}$ agree on a set of points having $b$ as a limit point. Let $\left\{x_{i}\right\}_{i=1}^{\infty}$ be a subset of such points converging to $b$. We then obtain that

$$
\left|\delta_{b}\right| \cdot \alpha_{b}\left(x_{i}\right)= \pm s_{\delta}\left(x_{i}\right)= \pm s_{0}\left(x_{i}\right)=0 \in \mathscr{H}_{x_{i}}^{n} .
$$

This contradicts the nondegeneracy of $\alpha_{b}$.

Sheaf cohomology with coefficients in a constant sheaf can be calculated as cohomology with coefficients in the module which is the stalk of the sheaf. Since we are interested in determining local properties of $B$, the following characterization is useful.

Proposition 1.2.2. The Leray sheaf in dimension $n$ is locally constant at $b \in B$ if, and only if, the $n$-winding function $\alpha_{b}$ is continuous at $b$.

Proof. $(\Rightarrow)$ Suppose that $\mathscr{l}^{n}=U \times \mathbf{Z}$, where $U$ is a neighborhood of $b$. Without loss of generality, we may assume that $U$ is connected and contained in the domain of $\alpha_{b}$ (i.e., the retraction $r_{b}$ is defined on $p^{-1}(U)$ ). Now $\alpha_{b}(y) \cdot 1_{y}$ is in a neighborhood of $1_{b}$ for $y$ sufficiently close to $b$; in particular, $\alpha_{b}(y)=1$ for all $y$ sufficiently close to $b$. Hence, $\alpha_{b}$ is continuous on a neighborhood of $b$.

$(\Leftarrow)$ Conversely, suppose that $\alpha_{b}$ is continuous on a connected neighborhood $U$ of $b$. Then $\alpha_{b}(y)=1$ for all $y$ in $U$. Let $\gamma_{b}$ be the selected generator of $\check{H}^{n}\left(p^{-1}(b)\right)$. Let $\gamma_{y}$ denote the element of $\check{H}^{n}\left(p^{-1}(y)\right)$ which is the image of $\gamma_{b}$ under the induced homomorphism $\left(r_{b} \mid p^{-1}(y)\right)^{*}$. Obviously, $\gamma_{y}$ generates $\breve{H}^{n}\left(p^{-1}(y)\right.$ ) (since $\gamma_{y}$ is $\alpha_{b}(y)$ times one of the generators of this stalk) and, for all $y$ in a small enough neighborhood $V$ of $b$ (for example, a neighborhood 
whose preimage over $p$ shape deforms into $p^{-1}(b)$ over $\left.U\right)$, the set $N=\left\{\gamma_{y} \mid\right.$ $y \in V\}$ forms a basic neighborhood of $\gamma_{b}$ in $\mathscr{H}^{n}$. Define a map $h:\left.\mathscr{H}^{n}\right|_{\pi(N)} \rightarrow$ $V \times \mathbf{Z}$ as follows: $i \cdot \gamma_{y} \mapsto(y, i)$. Then $h$ is a well-defined continuous map which is clearly bijective with continuous inverse. Thus, $\mathscr{H}^{n} \mid V$ is constant.

We can then apply the following Theorem of J. J. Walsh and J. Dydak to obtain the succeeding corollary.

Theorem [DyW]. Suppose $G$ is a finitely generated abelian group and $X$ is a complete metrizable space. If $S$ is a presheaf on $X$ such that

(1) each stalk $\mathscr{S}_{x}$ of the sheaf $\mathscr{S}$ generated by $S$ is isomorphic to $G$, and

(2) for each $x \in X$ and for each neighborhood $U$ of $x$ there exists a neighborhood $V \subset U$ of $x$ such that the image of $S(U) \rightarrow S(V)$ is finitely generated,

then $\mathscr{S} \mid W$ is locally constant for some open and dense subset $W$ of $X$.

Corollary 1.2.3. The Leray sheaf is locally constant in each dimension over a dense subset $W$ of $B$.

1.3. Sheaf cohomology and the Leray spectral sequence of a map. In this subsection, we give an extremely brief outline of the technical tools used in this work. There is no attempt to give detailed definitions of sheaf cohomology or spectral sequences, just the bare minimum required to orient the reader in the proofs of Theorems 1 and 2. For an easy transition into aspects of the theory, the reader is advised to peruse [A, DyW]; for all the details one should read $[\mathrm{Br} 1, \mathrm{Sw}, \mathrm{S}]$.

For a family of supports $\phi$ and sheaf $\mathscr{S}$ on the space $X$, the sheaf cohomology of $X$ with coefficients in $\mathscr{S}$ and supports in $\phi$, denoted $H_{\phi}^{*}(X ; \mathscr{S})$, is defined [Brl]. If $A \subset X$, then we write $H_{\phi}^{*}(A ; \mathscr{S})$ for $H_{\phi \mid A}^{*}\left(A ;\left.\mathscr{S}\right|_{A}\right)$. Sheaf cohomology of the pair $(X, A)$ can also be defined. For constant sheaves, the functor so defined agrees with the Alexander-Spanier theory in general and, in addition, agrees with the singular theory when $X$ is an ANR. Also, $\phi$ is elided from the expression if, and only if, $\phi$ is the family of closed supports on $X$.

For the pair $(X, A)$ we have the following long exact sequence:

$\cdots \rightarrow H_{\phi}^{q}(X, A ; \mathscr{S}) \rightarrow H_{\phi}^{q}(X ; \mathscr{S}) \rightarrow H_{\phi}^{q}(A ; \mathscr{S}) \rightarrow H_{\phi}^{q+1}(X, A ; \mathscr{S}) \rightarrow \cdots$.

If $X$ is a locally compact metric space, $\phi$ is the family of closed supports, and $x \in X$, then there is a natural isomorphism [Br1, p. 80]

$$
H_{x}^{*}(X ; \mathscr{S}) \cong H_{\phi}^{*}(X, X-x ; \mathscr{S}) \text {. }
$$

We need a corollary of the following theorem; our statement of it is not the most general, but is sufficient for our needs. The proof is found on p. 59 of [Br1].

Theorem 1.3.1. If $A$ is an open subset of $X$ and $\phi$ is the family of closed supports on $X$, then there is a natural isomorphism

$$
H_{\phi}^{*}(X, A ; \mathscr{S}) \cong H_{\phi \mid X-A}^{*}(X ; \mathscr{S}) .
$$


Lemma 1.3.2. Let $g$ be a compact subset of $X$. Then there is a natural isomorphism

$$
H_{\phi}^{*}(X, X-g ; \mathscr{S}) \cong H_{\phi \mid g}^{*}(X ; \mathscr{S}) .
$$

The following theorem is the foundation of our approach to Theorems 1 and 2; its proof is found in [ $\mathrm{Br} 1$, pp. 140-141].

Theorem 1.3.3 (Leray-Grothendieck). For a closed map $f: X \rightarrow Y$, there is a first quadrant spectral sequence

$$
E_{2}^{p, q}=H_{\phi}^{p}\left(Y ; \mathscr{H}^{q}[f]\right) \Rightarrow H_{\phi(\psi)}^{p+q}(X) .
$$

Remarks. (1) For our purposes we need only consider the family $\psi$ of closed supports on $X$, and either $\phi$ is the family of closed supports on $Y$ or $\phi=\{x\}$. In the former case $\phi(\psi)=\psi$, and in the latter $\phi(\psi)=\phi \mid \tilde{x}$, the closed subsets of $\tilde{x}$.

(2) $E_{r+1}^{p, q}=\operatorname{ker}\left(d_{r}\right) / \operatorname{im}\left(d_{r}\right)$, where the differential

$$
d_{r}^{p, q}: E_{r}^{p, q} \rightarrow E_{r}^{p+r, q-r+1}
$$

has bidegree $(r, 1-r)$.

(3) $E_{r}^{p, q}=E_{r+1}^{p, q}=E_{r+2}^{p, q}=\cdots=E_{\infty}^{p, q}$ for $r \geq p+q+1$.

(4) There is a filtration $0 \subset J_{0} \subset J_{1} \subset \cdots \subset J_{p}=H^{p}(X)$, where $J_{0}=E_{\infty}^{p, 0}$ and $J_{i} / J_{i-1}=E_{\infty}^{p-i, i}, i \leq p$.

(5) We also may use Čech sheaf cohomology for $\phi$ being closed supports [Sw].

(6) $[\mathrm{McC}]$ has a quick introduction concerning calculation of spectral sequences.

Note for the choice of supports in the latter of (1) above that, by Lemma 1.3.2,

$$
H_{\phi(\psi)}^{i}(X)=H^{i}\left(X, X-f^{-1}(x)\right) .
$$

We now give a relatively easy application of Theorem 1.3.3.

Lemma 1.3.4. Let $G$ be a decomposition of the complete metrizable space $M$ into subcontinua having the shape of closed, orientable n-manifolds. Let $A$ be an arc in $B=M / G$. Then there is a natural isomorphism $H^{1}\left(A ; \mathscr{H}^{n}\right) \cong H^{n+1}(\widetilde{A})$. Addendum. Suppose that $G$ is 1-acyclic. Then there is a natural isomorphism $H^{0}\left(A ; \mathscr{H}^{n}\right) \cong H^{n}(\widetilde{A})$.

Proof. We consider the map $f=\left.\pi\right|_{\tilde{A}}: \widetilde{A} \rightarrow A$, where $\pi$ denotes the decomposition map. Staring at the Leray-Grothendieck spectral sequence for $f$ brings one to the observation that $E_{2}^{p, q}=0$ for $q>n$; this is also true for $p>1$, since sheaf cohomology with paracompactifying supports respects dimension of a space [Sw, pp. 109-111].

In particular, $d_{i} \equiv 0$ for all $i \geq 2$. Thus,

$$
E_{2}^{p, q}=E_{\infty}^{p, q}
$$


for all $p$ and $q$. Considering the filtration of Remark (4) above, and observing that

$$
J_{n+1} / J_{n}=E_{\infty}^{0, n+1}=0
$$

it is clear that

$$
H^{n+1}(\widetilde{A})=J_{n+1}=J_{n}=E_{\infty}^{1, n}=E_{2}^{1, n}=H^{1}\left(A ; \mathscr{H}^{n}\right) .
$$

Now, we prove the addendum. Note that $n>1$. Suppose $G$ is 1 -acyclic. Then, by duality, $\mathscr{H}^{n-1}$ is the trivial sheaf on $A$. Thus, for $i<n$,

$$
J_{i} / J_{i-1}=E_{\infty}^{n-i, i}=E_{2}^{n-i, i}=0
$$

where the $J_{i}$ 's form the filtration of $H^{n}(\tilde{A})=J_{n}$. In particular,

$$
0=E_{2}^{n, 0}=E_{\infty}^{n, 0}=J_{0}=J_{n-1} .
$$

Finally, we calculate $H^{0}\left(A ; \mathscr{H}^{n}\right)=J_{n} / J_{n-1}=J_{n}=H^{n}(\widetilde{A})$.

We now apply 1.3 .4 to the case of $M$ being a manifold, in order to get two important lemmas.

Let $G$ be a codimension- $k$ manifold decomposition; let $B$ be the decomposition space and $p$ the decomposition map. Let

(1) $K=\bigcap\left\{K^{\prime} \subset B\left|\mathscr{H}^{n}\right|_{B-K^{\prime}}\right.$ is Hausdorff $\}$,

(2) $D=\left\{x \in B-K \mid \mathscr{H}^{n}\right.$ is not locally constant at $\left.x\right\}$,

(3) $C=\left\{x \in B \mid \mathscr{H}^{n}\right.$ is locally constant at $\left.x\right\}$, and

(4) $E=K \cup D$.

Note that $K, D$, and $C$ form a partition of $B$; that $K$ is closed, and $C$ is open, in $B$; and that $E$ is closed in $B$.

Lemma 1.3.5. Assume that $G$ and $M$ are as defined above. Let $A$ be an arc in $B$ such that

(a) $\partial A=\left\{d_{1}, d_{2}\right\} \subset D$,

(b) $A-\partial A \subset C$,

(c) $\alpha_{d_{i}}\left(c_{i}\right)=p_{i}$ for some $c_{i} \in A \cap C$ in the domain of $\alpha_{d_{i}}(i=1,2)$.

Then $H^{n+1}(\widetilde{A}) \cong \mathbf{Z}_{d}$, where $d$ is $\operatorname{gcd}\left(p_{1}, p_{2}\right)$ (the greatest common divisor of $\left.p_{1}, p_{2}\right)$.

Addendum. $H^{n}(\widetilde{A}) \cong \mathbf{Z}$, if $G$ is 1-acyclic.

Proof. By Lemma 1.3.4, it is enough to calculate $H^{1}\left(A ; \mathscr{H}^{n}\right)$. Note that $p_{1}, p_{2} \neq 0$. We do not prove here the simple case that at least one of the $p_{i}$ is equal to one, for we do not need the result. There are then two remaining cases to analyze.

Case 1. $d=p_{1}=p_{2}$. We define a sheaf map from the constant sheaf $A \times \mathbf{Z}$ to $\left.\mathscr{H}^{n}\right|_{A}$. Now, $\left.\mathscr{H}^{n}\right|_{A-\left\{d_{1}, d_{2}\right\}}$ is constant, so for $c \in A-\left\{d_{1}, d_{2}\right\}$ pick elements $\gamma_{c}$ of the stalk $\mathscr{H}_{c}^{n}$ so that $\gamma_{c}$ corresponds to $p$ under the map; we can extend 
the map to $A \times \mathbf{Z}$ by picking the generator $\gamma_{d_{i}}$ of $\mathscr{H}_{d_{i}}^{n}$ that corresponds under the defining retraction to $p_{i} \cdot \gamma_{c}$. Obviously this map is an injection; let $\mathscr{Q}$ denote the quotient sheaf. We thus have the following short exact sequence of sheaves:

$$
0 \rightarrow A \times\left.\mathbf{Z} \rightarrow \mathscr{H}^{n}\right|_{A} \rightarrow \mathscr{Q} \rightarrow 0 .
$$

As a consequence we get a long exact sequence

$$
\cdots \rightarrow H^{i}(A) \rightarrow H^{i}\left(A ; \mathscr{H}^{n}\right) \rightarrow H^{i}(A ; \mathscr{Q}) \rightarrow \cdots .
$$

Since $H^{1}(A)=0=H^{2}(A)$, to calculate $H^{1}\left(A ; \mathscr{H}^{n}\right)$ we need only calculate $H^{1}(A ; \mathscr{Q})$.

Since $p_{1}=p_{2}$, we have

$$
\mathscr{Q}_{b}= \begin{cases}0, & \text { for } b=d_{1}, d_{2}, \\ \mathbf{Z}_{d}, & \text { otherwise. }\end{cases}
$$

Moreover, this sheaf is constant over the interior of $A$. In fact the topology of the sheaf is the same as taking the constant sheaf $A \times \mathbf{Z}_{d}$, restricting it to the interior of the arc, and then extending the sheaf so it is trivial over the endpoints of the arc. So $H^{1}(A ; \mathscr{Q})=H^{1}\left(A,\left\{d_{1}, d_{2}\right\} ; \mathbf{Z}_{d}\right)=\mathbf{Z}_{d}$.

Case 2. $p_{1} \neq p_{2}$. Note in the argument of the first case that if, say, the endpoint $d_{2}$ of $A$ were also not in $D \cup K$, then $H^{n+1}(\widetilde{A})=0$. We use this observation in conjunction with the Mayer-Vietoris sequence to calculate the cohomology in the second case.

Let $l$ be the least common multiple of $p_{1}$ and $p_{2}$. Pick $c \in A-\partial A$. Let $A_{i}$ denote the closure in $A$ of the component of $A-\{c\}$ containing $d_{i}$. Applying the cohomology version of the Mayer-Vietoris sequence for the pair $\left(\tilde{A}_{1},{\widetilde{A_{2}}}_{2}\right)$ we obtain

$$
0 \rightarrow H^{n}(\widetilde{A}) \rightarrow \sum_{i=1}^{2} H^{n}\left(\widetilde{A_{i}}\right) \rightarrow H^{n}(\tilde{c}) \rightarrow H^{n+1}(\widetilde{A}) \rightarrow 0 .
$$

So applying the above note to $A_{i}$ we have

$$
0 \rightarrow H^{n}(\widetilde{A}) \rightarrow \mathbf{Z} \oplus \mathbf{Z} \rightarrow \mathbf{Z} \rightarrow H^{n+1}(\widetilde{A}) \rightarrow 0 .
$$

In the middle map, it is clear that the pairs $(1,0)$ and $(0,1)$ are mapped to $p_{1}$ and $p_{2}$, respectively. The ideal generated by $\left\{p_{1}, p_{2}\right\}$ is the principal ideal $(d)$. Thus, $H^{n+1}(\tilde{A})$, the image of the last map, is isomorphic to $\mathbf{Z} /(d)=\mathbf{Z}_{d}$.

The addendum's proof is trivial upon observing that $H^{n}(\widetilde{A}) \cong H^{0}\left(A ; \mathscr{H}^{n}\right)$. It is clear that the latter group is $\mathbf{Z}$.

Lemma 1.3.6. Assume that $G$ and $M$ are as in 1.3.5. Let $A$ be an arc in $B$ such that

(a) $\partial A=\left\{k_{1}, k_{2}\right\} \subset K$,

(b) $A-\partial A \subset C$,

(c) $\alpha_{k_{t}}\left(c_{i}\right)=0$ for some $c_{i} \in A \cap C$ in the domain of $\alpha_{k_{t}}(i=1,2)$.

Then $H^{n+1}(\widetilde{A}) \cong \mathbf{Z}$. 
Proof. Using 1.3.4 as in the proof above, we need only calculate $H^{1}\left(A ; \mathscr{C}^{n}\right)$.

Choose $c \in A-\partial A$. Let $A_{i}$ denote the closure of the component of $A-\{c\}$ containing $k_{i}(i=1,2)$. Apply the Mayer-Vietoris sequence (coefficients in $\left.\mathscr{H}^{n}\right)$ to the triple $\left(A, A_{1}, A_{2}\right)$, to see that

$$
0 \rightarrow \mathbf{Z} \rightarrow \check{H}^{1}\left(A ; \mathscr{H}^{n}\right) \rightarrow \check{H}^{1}\left(A_{1} ; \mathscr{H}^{n}\right) \oplus \check{H}^{1}\left(A_{2} ; \mathscr{H}^{n}\right) \rightarrow 0 .
$$

Thus it suffices to show that $\check{H}^{1}\left(A_{1} ; \mathscr{H}^{n}\right)$ is trivial. To prove this choose a nested collection of subarcs $\varpi_{1}, \varpi_{2}, \ldots$ of $A_{1}=\varpi_{0}$ whose intersection is $k_{1}$. The inclusion-induced homomorphism

$$
\check{H}^{1}\left(A_{1} ; \mathscr{H}^{n}\right) \rightarrow \check{H}^{1}\left(\varpi_{j} ; \mathscr{H}^{n}\right)
$$

is obviously an isomorphism for all $j$. Thus, using continuity of sheaf cohomology [Brl, p. 71], $0=\check{H}^{1}\left(k_{1} ; \mathscr{H}^{n}\right)=\check{H}^{1}\left(\varliminf^{\lim } \varpi_{j} ; \mathscr{H}^{n}\right)=\varliminf_{\longrightarrow} \check{H}^{1}\left(\varpi_{j} ; \mathscr{H}^{n}\right) \cong$ $\check{H}^{1}\left(A_{1} ; \mathscr{H}^{n}\right)$.

Hence, by Lemma 1.3.4, $\mathrm{Z} \cong \check{H}^{1}\left(A ; \mathscr{H}^{n}\right) \cong \check{H}^{n+1}(\widetilde{A})$.

1.4. Properties of generalized manifolds and ANRs. In this subsection we define and discuss crucial properties of generalized manifolds and ANRs. Theorem 1.4.5 and Corollary 1.4.12 are especially important for the investigations of $\S \S 2$ and 3. For details about proofs of the deep results, the reader is referred to [Hu, W, Br1, Br2, DyW, A, D3]. Throughout this section $X$ is assumed to be a locally compact metrizable space.

Definitions. $X$ is a generalized $k$-manifold over $\mathbf{Z}$, abbreviated as $k-\mathrm{gm}_{\mathbf{Z}}$ or $k$-gm, if

(1) $X$ is a finite-dimensional ANR and

(2) for all $x \in X$,

$$
H_{i}(X, X-x)= \begin{cases}0, & \text { for } i \neq k, \\ \mathbf{Z}, & \text { for } i=k .\end{cases}
$$

The orientation sheaf $\mathscr{O}$ of a $k$-gm is generated by the presheaf

$$
U \mapsto H_{k}(X, X-U) .
$$

If $\mathscr{O}$ is (locally) constant, then we say $X$ is (locally) orientable; otherwise $X$ is nonorientable.

Remark. Item (1) (and the choice of coefficient ring) of our definition of $k$-gm is less general than the local cohomological connectivity and finite cohomological dimension posited in the "classical" definition (see [Br2]); many of the results that follow were proved in the more general setting.

The following theorem is a rather deep result. It was first proved by G. Bredon in [Br2]; it also follows as a corollary of a much more general result of J. Dydak and J. J. Walsh [DyW]. 
Theorem 1.4.1 (Bredon). Every $k$-gm is locally orientable.

Corollary 1.4.2. Every simply-connected $k$-gm is orientable.

Corollary 1.4.3. Every nonorientable $k$-gm $X$ has an orientable double cover $\widetilde{X}$.

For orientable $k$-gm's there is an Alexander duality theorem (see [A] or [W]). This will be quite useful to us in the characterizations of $\S \S 2.2$ and 3.2.

Theorem 1.4.4. Let $X$ be an orientable k-gm. Let $B \subset A$ be closed subsets of $X$. Then there is a natural isomorphism

$$
D: H_{i}(X-B, X-A) \cong H_{c}^{k-i}(A, B) .
$$

In particular, the following diagram commutes:

$$
\begin{aligned}
& \cdots \rightarrow H_{c}^{k-i}(X, A) \rightarrow H_{c}^{k-i}(X, B) \rightarrow H_{c}^{k-i}(A, B) \quad \rightarrow \cdots \\
& \uparrow \cong \quad \uparrow \cong \quad \uparrow \\
& \cdots \rightarrow H_{i}(X-A) \rightarrow H_{i}(X-B) \rightarrow H_{i}(X-B, X-A) \rightarrow \cdots
\end{aligned}
$$

Using the local duality of a $k$-gm and a Mayer-Vietoris argument, one can show the following

Theorem 1.4.5. Let $A$ be an arc in a k-gm $X$. Then

$$
H_{i}(X, X-A)= \begin{cases}0, & \text { for } i \neq k, \\ \mathbf{Z}, & \text { for } i=k .\end{cases}
$$

We are now finished with our discussion of $k$-gm's and turn our attention to properties of ANRs. To start with, we define some connectivity properties.

Definition. A space $X$ is locally connected in dimension $k$, denoted $k$-LC, if for every $x \in X$ and neighborhood $U$ of $x$ in $X$ there is a neighborhood $V$ of $x$ in $U$ with

$$
\pi_{k}(V) \rightarrow \pi_{k}(U)
$$

trivial. If $X$ is $k$-LC for every $k \leq i$ (resp. $k<\infty$ ), we say $X$ is $\operatorname{LC}^{i}$ (resp. $\mathrm{LC}^{\infty}$ ). Moreover, we say $X$ is $\mathrm{LC}^{*}$ if $X$ is locally contractible

Definition. Let $\mathscr{S}$ be a sheaf on $X$ with Z-module stalks. Then we say $X$ is cohomologically locally connected in dimension $i$ with respect to $\mathscr{S}$, abbreviated $i$-clc ${ }_{\mathscr{S}}$, at $x$ if for all neighborhoods $U$ of $x$ there is a neighborhood $V \subset U$ of $X$ so that the image of the inclusion-induced map

$$
\check{H}^{i}(U ; \mathscr{S}) \rightarrow \check{H}^{i}(V ; \mathscr{S})
$$

is trivial as a $\mathbf{Z}$-module. If the above property holds for all $x \in X$ then we say

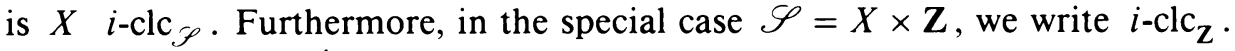

Finally, $X$ is $\operatorname{clc}_{\mathscr{P}}^{k}$ (resp. $\operatorname{clc}_{\mathscr{P}}^{\infty}$ or $\operatorname{clc}_{\mathscr{P}}$ ) at $X$ if $X$ is $i$-clc $\mathscr{\mathscr { P }}$ at $x$ for all $i \leq k<\infty$ (resp. $i<\infty)$. If this is true for all $x \in X$, then we drop the phrase "at $x$." A subscript of $\mathbf{Z}$ again indicates the trivial sheaf $\mathscr{S}=X \times \mathbf{Z}$. 
Remarks. (1) The Alexander-Spanier and the Čech cohomology theories are isomorphic for paracompact Hausdorff spaces [S, Corollary 8, p. 334].

(2) There are corresponding definitions for homology, denoted $i$-hlc, hlc ${ }_{\infty}^{k}$, etc. [Br1, p. 253]; for these we will use the Alexander-Spanier homology functor. The following lemma is of use to us in proving Lemma 1.4.8.

Lemma 1.4.6. Let $X$ be a metric space which is $c l c \equiv c_{c}^{\infty}$. Then $X$ is hlc $\equiv$ $h l c_{\mathbf{Z}}^{\infty}$.

Sketch of the proof. Given $k \geq 0, x \in X$, select neighborhoods $W \subset V \subset U$ of $x$ in $X$ so that the maps induced by inclusion on the cohomologies are trivial in dimensions $k$ and $k+1$. Apply the categorically natural short exact sequence of the Universal Coefficient Theorem for Alexander-Spanier cohomology [S, $\mathrm{p}$. $244]$ to these maps and chase the resulting diagram to see that $H_{k}(W)$ dies in $H_{k}(U)$.

The following proposition, an extension of the Hurewicz theorem [Hr], appears as Theorem 3.2 in [DyS2].

Proposition 1.4.7 (Dydak-Segal). Suppose $X$ is an $L C^{k}(k \geq 1)$ complete metrizable space such that for each $x \in X$ and for each neighborhood $U$ of $x$ there exists a neighborhood $V$ of $x$ in $U$ with the composition of homomorphisms

$$
\pi_{k+1}(V, x) \rightarrow H_{k+1}(V) \rightarrow \check{H}_{k+1}(V) \rightarrow \check{H}_{k+1}(U)
$$

trivial. Then $X$ is $L C^{k+1}$.

The following lemma is a corollary of 1.4 .7 and 1.4.6, using induction.

Lemma 1.4.8. Suppose $X$ is a complete metrizable space which is $L C^{1}$ and clc. Then $X$ is $L C^{\infty}$.

The following corollary has been the goal of the previous two pages.

Corollary 1.4.9. If $X$ is a finite-dimensional $L C^{1}$, clc metric space then $X$ is an $A N R$.

The following theorem is found in [DyS2]; we use it to establish a useful criterion for determining if the decomposition space of a codimension- $k$ manifold decomposition is an ANR.

Theorem 1.4.10 (Dydak-Segal). Let $f: X \rightarrow Y$ be a proper surjection of locally compact spaces whose point inverses are FANRs. If $X$ is an ANR and $f$ has the $A H L P$ with respect to all $j$-cells, $0 \leq j \leq k$, then $Y$ is $L C^{k+1}$.

Remarks. (A) A finite-dimensional compactum is an FANR if it has the shape of an ANR; see [DyS1, pp. 74-75].

(B) A map $f: X \rightarrow Y$ between locally compact metrizable spaces has the approximate homotopy lifting property $(A H L P)$ with respect to the space $Z$ if, 
given any open cover $\mathscr{U}$ of $Y$ and commutative diagram

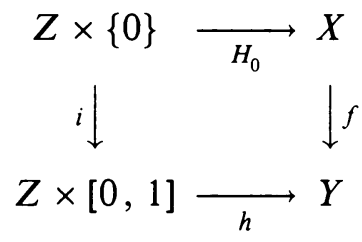

where $i$ denotes the obvious inclusion, there is an extension $H: Z \times[0,1] \rightarrow X$ of $H_{0}$ so that for any $(z, t)$ there is an element $U$ of $\mathscr{U}$ containing both $(f \circ H)(z, t)$ and $h(z, t)$.

A map is an approximate fibration if it has the A.HLP with respect to all metrizable spaces; this concept was developed in [CD1].

Corollary 1.4.11. Let $G$ be a codimension- $k$ manifold decomposition of the manifold $M^{n+k}$. Then $B$ is $L C^{1}$.

Corollary 1.4.12. Assume the hypotheses of 1.4 .11 and, in addition, that $\operatorname{dim} B \leq$ $d<\infty$. Then the following statements are equivalent:

(1) $B$ is an $A N R$.

(2) $B$ is $\operatorname{clc}_{\mathbf{Z}}^{\infty}$.

(3) $B$ is $\operatorname{clc}_{\mathrm{Z}}^{d}$.

1.5. Potpourri. We include in this subsection various algebraic and sheaf theoretic results needed in the sequel, in order to retain clarity. The first is a special result about torsion $\mathbf{Z}$-modules used in the proof of Theorem 2 exactly once. The second is a sort of "zig-zag" lemma needed in our discussion of local connectivity propeities of the decomposition space. The third gives some sufficient conditions for which a sheaf that is locally constant off a certain closed set is actually constant off that set, needed in $\S 3$. The last is a calculation used four times in the sequel.

The first lemma is used in $\S 2$ to show that the module of extensions of a certain group by $\mathbf{Z}$ is not finitely generated. The proof is simple, and uses the following elementary proposition.

Proposition [H, p. 222]. Let $A$ be a torsion module over a UFD R. Let

$$
A(p)=\{a \in A \mid \text { a has order of some power of } p\}
$$

for any prime $p \in R$. Then $A=\sum A(p)$, with the sum being taken over all primes $p \in R$.

Lemma 1.5.1. Suppose $A_{i}$ are $\mathbf{Z}$-modules for $i=1,2, \ldots$, and there exist surjections $\psi_{i}: A_{i} \rightarrow A_{i+1}$ with kernel isomorphic to $\mathbf{Z}_{p_{i}}$, where $\left\{p_{i} \mid p_{i}>\right.$ $1, i=1,2,3, \ldots\}$ is a list of (not necessarily distinct) positive integers. If the direct limit of the system $\left\{\left(A_{i}, \psi_{i}\right)\right\}$ is trivial, then $A_{1}=\sum A_{1}(p)$. Moreover, either there is a prime $p$ such that $A_{1}(p)$ is countably infinitely generated or $A_{1}(p)$ is nontrivial for infinitely many primes $p$. 
Proof. Let $K_{i}$ denote the kernel of $\theta_{i} \equiv \psi_{i} \circ \psi_{i-1} \circ \cdots \circ \psi_{2} \circ \psi_{1}$. Obviously, $K_{i}$ is a proper subset of $K_{i+1}$. By hypothesis $K_{1}$ is isomorphic to $\mathbf{Z}_{p_{1}}$. By induction we see that each $K_{i}$ is a finitely generated torsion module; moreover, $A_{1}$ is the countable nested union of the $K_{i}$ since the direct limit is trivial. Therefore, $A_{1}$ has countably infinite cardinality and is (as is each $A_{i}$ ) a torsion $\mathbf{Z}$-module. Since finitely generated torsion $\mathbf{Z}$-modules have finite cardinality, $A_{1}$ is generated as a $\mathbf{Z}$-module by an infinitely countable set.

By the proposition above, $A_{1}=\sum A_{1}(p)$, with the sum being taken over all primes $p \in \mathbf{Z}$.

Note that for each prime $p, A_{1}(p)$ is necessarily countably generated. Suppose there is no prime $p$ such that $A_{1}(p)$ is countably infinitely generated. Then, for each prime $p \in \mathbf{Z}, A_{1}(p)$ is finitely generated and, hence, a finite set. Thus, $A_{1}(p)$ must be nontrivial for infinitely many primes, since $A_{1}$ is infinite.

The following theorem is often useful. The proof is straightforward and may be found in the references cited.

Theorem 1.5.2 [ $\mathrm{Br} 2$, p. 77, DyW]. Let the following diagram denote a commutative diagram of $\mathbf{Z}$-modules, where each of the vertical maps $\rho, \sigma$ has finitely generated image, and the middle row is exact. Then the image of $L_{1}$ under the map $\tau_{2} \circ \tau_{1}$ is finitely generated.

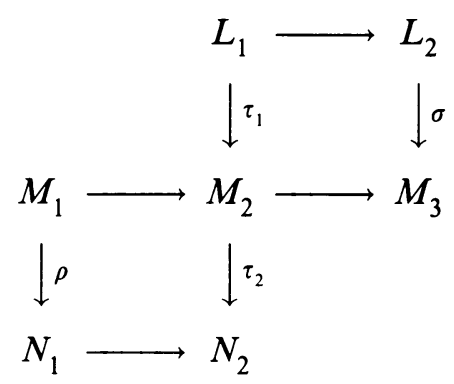

The following lemma is applied at certain crucial instances. Some of the hypotheses may be weakened further, but, as this is not necessary for the results obtained in this paper, we leave such generalization for the interested reader.

Lemma 1.5.3. Suppose $U$ is a metrizable ANR, and $V$ is a subset of $U$. Suppose that $D \subset U$ is such that $H^{1}(U-\bar{D}) \rightarrow H^{1}(V-\bar{D})$ has trivial image. Furthermore, assume $\mathscr{S}$ to be a sheaf on $U$ with stalks isomorphic to $\mathbf{Z}$ which is locally constant on $U-\bar{D}$. Then $\mathscr{S}$ is constant on $V-\bar{D}$.

Proof. Let $U^{\prime}=U-\bar{D}$ and $V^{\prime}=-V-\bar{D}$. First note that $\left.\mathscr{S}\right|_{U^{\prime}}$, being locally constant, can be extended to a line bundle $\zeta$ over $U^{\prime}$. Using the fact that $H^{1}\left(U^{\prime}\right) \rightarrow H^{1}\left(V^{\prime}\right)$ has trivial image, it can be easily shown that the characteristic class $\omega_{1}$ of $\left.\zeta\right|_{V^{\prime}}$ is 0 . Thus, $\left.\zeta\right|_{V^{\prime}}$ is orientable [Hus, p. 246]. Orientable line bundles are trivial line bundles. Hence, $\left.\mathscr{S}\right|_{V^{\prime}}$ is constant. 
Lemma 1.5.4. Let $g_{1}, g_{2}$ denote orientable closed $n$-manifolds and let $f: g_{1} \rightarrow$ $g_{2}$ be a map which is an injection with respect to the global orientation class $\zeta_{1} \in H_{n}\left(g_{1}\right)$, say $f_{*}\left(\zeta_{1}\right)=k \cdot \zeta_{2}$. Let $j=\min \left\{i \mid \widetilde{H}_{i}\left(g_{2}\right) \neq 0\right\}$. Then $f^{*}$ : $H^{j}\left(g_{2}\right) \rightarrow H^{j}\left(g_{1}\right)$ is an injection.

Proof. First note that $j>0$ and, by the Universal Coefficient Theorem, $H^{j}\left(g_{2}\right)$ is free. Choose $\xi \in H^{j}\left(g_{2}\right)$. Now we apply [S, 16, p. 254]. Namely,

$$
f_{*}\left(f^{*} \xi \frown \zeta_{1}\right)=\xi \frown f_{*} \zeta_{1}=\xi \frown k \cdot \zeta_{2}=k \cdot\left(\xi \frown \zeta_{2}\right) .
$$

Note $H_{n-j}\left(g_{2}\right) \cong H^{j}\left(g_{2}\right)$ is free; so, if $\xi \neq 0$, then $k \cdot\left(\xi \frown \zeta_{2}\right)$ is nonzero. Therefore, $f^{*}$ is an injection.

Lemma 1.5.5. Let $G$ be a j-acyclic shape manifold decomposition of the $l$ manifold $M(j<l), B=M / G$, and $p$ the decomposition map. Let $U$ be a connected, $j-(j+1)-h l_{\mathbf{Z}}$ neighborhood of $x \in B$ such that $\widetilde{U}=p^{-1}(U)$ is orientable. Let $W \subset V \subset U$ be connected neighborhoods of $x$ such that $\widetilde{V}$ shape deforms to $\tilde{x}$ in $\widetilde{U}, \widetilde{W}$ shape deforms to $\tilde{x}$ in $\widetilde{V}$, and so that the inclusions of $W$ into $V$ and $V$ into $U$ are homologically trivial in dimensions $j, j+1$. Let $A$ be an arc in $W-\{x\}$, with $\check{H}^{l-j-1}(\widetilde{A})=N$, a cyclic Z-module. Then $H_{j}(V, V-A) \cong N$.

Proof. Note that

$$
N \cong \check{H}^{l-j-1}(\widetilde{A}) \cong H_{j+1}(\widetilde{U}, \widetilde{U}-\tilde{A}),
$$

the latter map given by Alexander duality; and similarly for " $U$ " replaced by " $V$ " or " $W$."

We consider following the commutative diagram with exact rows:

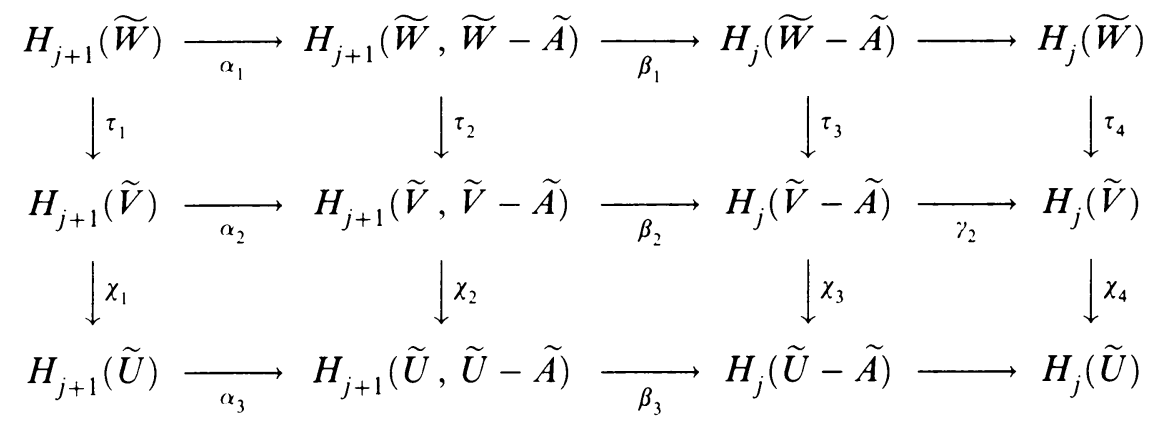

Note that $\chi_{2}, \tau_{2}$ are excision isomorphisms and that $\chi_{4}, \tau_{4}$ are zero maps. The latter fact implies that the image of $\tau_{3}$ lies in the image of $\beta_{2}$. It can also be seen that, since $A$ misses $x$ and $\tilde{x}$ carries the image of $\tau_{1}, \chi_{1}$, the maps preceding $\alpha_{1}, \alpha_{2}$ in the long exact sequence are surjections and, hence, the maps $\beta_{1}, \beta_{2}$ are injective; the image of each is $N$. Note that $\left.\tau_{3}\right|_{\text {im } \beta_{1}}$ is injective. Thus, the image of $\tau_{3}$ is nontrivial and contains $N$. But im $\tau_{3} \subset$ $\operatorname{ker} \gamma_{2}=\operatorname{im} \beta_{2}=N$. Thus, we must have the image of $\tau_{3}$ isomorphic to $N$. 
Using the Vietoris-Begle Theorem, we conclude that

$$
H_{j}(W-A) \rightarrow H_{j}(V-A)
$$

has image isomorphic to this module.

Finally, we consider

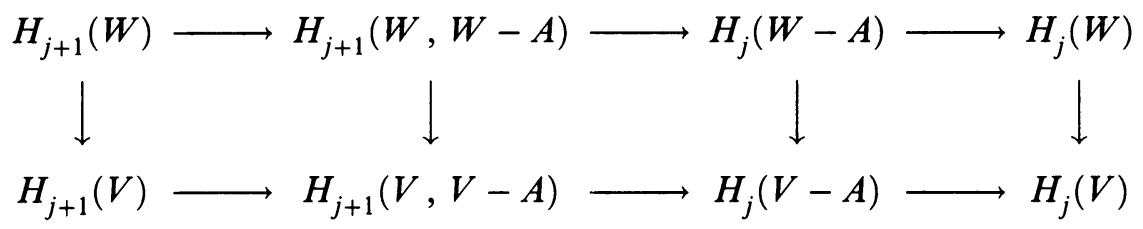

The second vertical arrow is an excision isomorphism, the first and fourth vertical arrows are trivial maps, by hypothesis. The conclusion now follows.

\section{HOMOLOGY SPHERE DECOMPOSITIONS IN THE MIDDLE DIMENSION}

This section contains the proof of Theorem 1 and its consequences. The following are the standing hypotheses for this section. $M$ is an $(n+k)$-manifold, $k>2, G$ an upper semicontinuous decomposition (uscd) of $M$ into shape homology $n$-spheres, $n \geq 2, p$ the decomposition map, and $B$ the decomposition space. For $A \subseteq B$, let $\widetilde{A}$ denote $p^{-1}(A)$. Furthermore, we assume that $\operatorname{dim} B<\infty$ and that $E=D \cup K$ does not locally separate $B$.

In $\S 2.1$, we find there are long exact sequences for $p$, and use these to determine immediately discernible local properties of $B$. Under the hypotheses of Theorem 1 it is shown in $\S 2.2$ that $B$ being a $k$-gm is equivalent to the discontinuity set $E=D \cup K$ being locally finite; this is used frequently in $\S 2.3$, which contains the proof of Theorem 1.

2.1. The long exact sequences of a homology sphere decomposition. The following lemmas compute Gysin sequences [Br, p.151] from the Leray spectral sequence.

Lemma 2.1.1. For all sets $U \subseteq B$ there is a long exact sequence

$$
\cdots \rightarrow H^{i}(U ; \mathbf{Z}) \rightarrow H^{i}(\widetilde{U} ; \mathbf{Z}) \rightarrow H^{i-n}\left(U ; \mathscr{H}^{n}\right) \rightarrow \cdots
$$

which is natural with respect to inclusion maps.

Lemma 2.1.2. For all compact subsets $A$ of $B$ and sets $U$ containing $A$, there is a long exact sequence

$$
\cdots \rightarrow H^{i}(U, U-A) \rightarrow H^{i}(\widetilde{U}, \widetilde{U}-\tilde{A}) \rightarrow H^{i-n}\left(U, U-A ; \mathscr{C}^{n}\right) \rightarrow \cdots
$$

which is natural with respect to inclusion maps of neighborhoods of $A$.

Proof of 2.1.1 and 2.1.2. Note that for each stalk

$$
\mathscr{H}^{q}[p]_{x}= \begin{cases}0, & q \neq 0, n, \\ \mathbf{Z}, & q=0, n,\end{cases}
$$


and, therefore, in the Leray spectral sequence for the map $p$ we have $E_{2}^{p, q}=0$ for $q \neq 0, n$. From a well-known lacunary property of spectral sequences (see [McC, pp. 6-9]), we obtain a long exact sequence

$$
\cdots \rightarrow E_{2}^{i, 0} \rightarrow H_{\phi(\psi)}^{i}(\widetilde{U}) \rightarrow E_{2}^{i-n, n} \rightarrow E_{2}^{i+1,0} \rightarrow \cdots .
$$

Lemma 2.1.1 follows by taking the family of supports $\phi$ to be the closed subsets of $U$. Taking $\phi$ to be the family consisting of $\phi \mid A$ and applying Lemma 1.3.1 proves 2.1.2.

The following is useful in investigating local properties of $B$.

Proposition 2.1.3. Given $b \in B$ and $i \in \mathbf{Z}$, the following are equivalent:

(1) $B$ is $i-c l c_{\mathbf{Z}}$ at $b$.

(2) $B$ is $(i-n-1)-c c_{\mathscr{P}^{n}}$ at $b$.

Proof. Assume $b \in B$ and $U$ a neighborhood of $b$ so that $\widetilde{U}$ shape retracts to $\tilde{b}$. Let $V$ be a neighborhood of $b$ contained in $U$ so that $\widetilde{V}$ [shape] deforms in $\widetilde{U}$ to $\tilde{b}$. Let $W$ be a neighborhood of $b$ in $V$ so that $\widetilde{W}$ [shape] deforms to $\tilde{b}$ in $\tilde{V}$. In particular,

$$
\text { image }\left\{H^{j}\left(\widetilde{A}_{1} ; \mathbf{Z}\right) \rightarrow H^{j}\left(\widetilde{A}_{2} ; \mathbf{Z}\right)\right\}=\left\{\begin{array}{lc}
0, & j \neq 0, n, \\
\mathbf{Z}, & j=0, n,
\end{array}\right.
$$

where $\left(A_{1}, A_{2}\right)$ is either of the pairs $(U, V)$ or $(V, W)$.

We also have

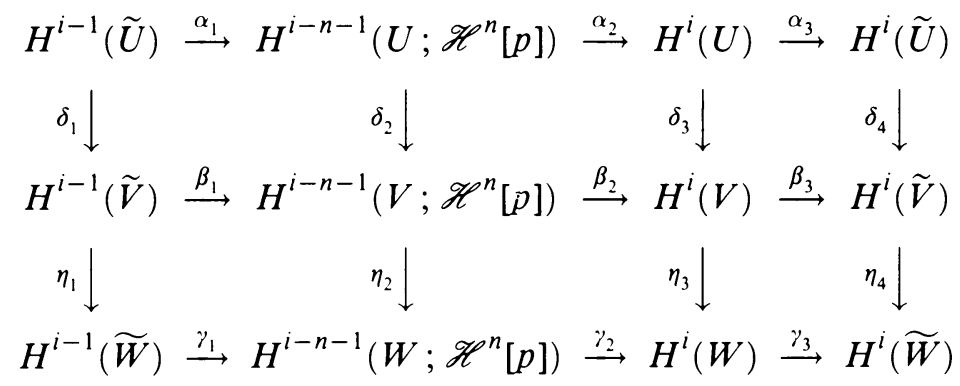

$(1) \Rightarrow(2)$ Without loss of generality, we may assume that image $\delta_{3}$ is finitely generated.

Now we apply Theorem 1.5.2 to the following portion of the diagram:

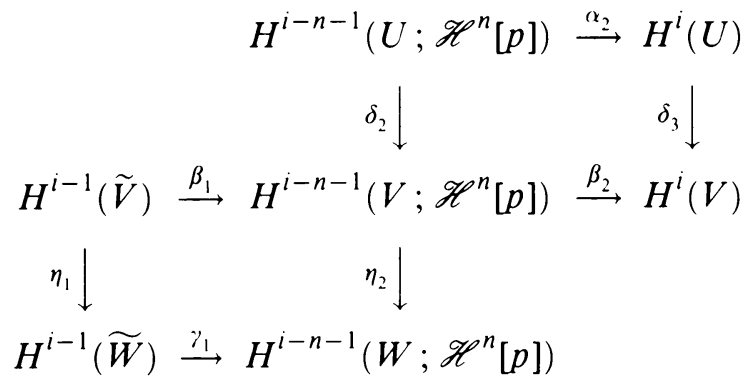


$(2) \Rightarrow(1)$ Without loss of generality, we may assume that image $\eta_{2}$ is finitely generated.

We apply Theorem 1.5.2 to the following portion of the diagram:

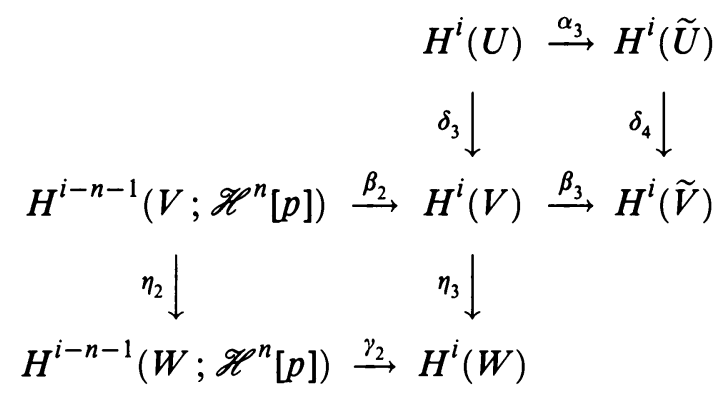

Corollary 2.1.4. $B$ is $c l c_{\mathrm{Z}}^{n}$ at all points.

Proof. Since for $i \leq n$ we get $H^{i-n-1}\left(U ; \mathscr{H}^{n}\right)=0$ trivially, the result follows from the previous proposition.

Corollary 2.1.5. If the Leray sheaf in dimension $n(n>2)$ is locally constant at each $b \in B$, then $B$ is a $k$-gm.

Proof. First we show that $B$ is an ANR, then that $B$ has the correct local relative homology.

Pick $b \in B$. Let $U$ be a neighborhood of $b$ over which $\mathscr{H}^{n}$ is constant, so $\mathscr{H}^{n} \cong U \times \mathbf{Z}$. Then for all $A \subseteq U, H^{i}\left(A ; \mathscr{H}^{n}\right) \cong H^{i}(A ; \mathbf{Z})$. By Corollary 2.1.4, pick a neighborhood $V_{1} \subset U$ of $b$ so that for all $i \leq n$

$$
\text { image }\left\{H^{i}(U) \rightarrow H^{i}\left(V_{1}\right)\right\}
$$

is trivial. By Proposition 2.1.3 we obtain that $B$ is $\operatorname{clc}_{\mathbf{Z}}^{2 n}$ at $b$. Continuing inductively, we find a neighborhood $V_{i} \subset U$ of $b$ to get that $B$ is $\operatorname{clc}_{\mathrm{Z}}^{n \cdot 2^{i}}$ at $b$. When $i$ is such that $n \cdot 2^{i}$ exceeds $\operatorname{dim} B$, then we may conclude that $B$ is $\operatorname{clc}_{\mathbf{Z}}^{\infty}$ at $b$. Since $b$ was arbitrarily chosen, $B$ is clc. Now apply 1.4.12.

This concludes the first step.

We now show that

$$
H^{i}(B, B-b)= \begin{cases}0, & i \neq k, \\ \mathbf{Z}, & i=k .\end{cases}
$$

It is an exercise in dimension theory (first see [N, p. 206]) that for $i>$ $\operatorname{dim} B, H^{i}(B, B-b)=0$ (consider $B-b$ as a direct limit of complements of neighborhoods of $b$ ).

Pick a neighborhood $U$ of $b$ so that $\widetilde{U}$ is an orientable submanifold of $M$ (this is possible since the elements have the shape of homology $n$-spheres and $n \geq 2$ ). Then using the Universal Coefficient Theorem and Alexander duality we obtain

$$
H^{i}(\tilde{U}, \tilde{U}-\tilde{b})= \begin{cases}0, & i \neq k, n+k, \\ \mathbf{Z}, & i=k, n+k,\end{cases}
$$




$$
H_{i}(\tilde{U}, \tilde{U}-\tilde{b}) \cong H^{n+k-i}(\tilde{b})= \begin{cases}0, & i \neq k, n+k, \\ \mathbf{Z}, & i=k, n+k .\end{cases}
$$

By this and the long exact sequence of Lemma 2.1.2 we get

$$
H^{i}(U, U-b) \cong H^{i-n-1}(U, U-b),
$$

for $i<k, k+1<i<n+k$, and $n+k+1<i$. Arguing as in the first step, we may conclude, for $i<k, k<i<n+k$, and $i \geq n+k$, that $H^{i}(U, U-b)=0$ (the case $i=n+k$ follows from dimension theory).

For $i=k$ we get (from the same long exact sequence)

$$
0 \rightarrow H^{k}(U, U-b) \rightarrow \mathbf{Z} \rightarrow H^{k-n}(U, U-b)=0 .
$$

Thus, $H^{k}(U, U-b) \cong \mathbf{Z}$.

Thus, we have shown $B$ to be a generalized cohomology manifold. Now apply the Universal Coefficient Theorem [S, p. 248].

Corollary 2.1.6. The set of points $\mathscr{G}$ at which $B$ is a $k$-gm contains an open dense subset $C$ of $B$.

Proof. Let $C \subset B$ be the open dense subset of $B$ on which the sheaf is locally constant. Then $\Omega=\widetilde{C}$ is an open submanifold of $M$. Apply Corollary 2.1.5 to $\left.p\right|_{\Omega}: \Omega \rightarrow C$. So, $C$ is a $k$-gm.

The following application is also a corollary of Proposition 3.1 in [DW3].

Corollary 2.1.7. If $k<n$, then $B$ is a $k$-gm.

Proof. Since $\operatorname{dim} B \leq k<n<\infty$ and $B$ is, by $2.1 .4, \operatorname{clc}_{\mathbf{Z}}^{k}$ (hence clc), it follows from 1.4.12 that $B$ is an ANR.

All that remains to be shown is that for all $b \in B$

$$
H_{j}(B, B-b)= \begin{cases}0, & j \neq k \\ \mathbf{Z}, & j=k .\end{cases}
$$

By the proof of the latter half of Corollary 2.1.5, we have $H^{i}(B, B-b)=0$ for $i<k$; the dimension of $B$ ensures that the same is true for $i>k$. Finally, examining the long exact sequence of 2.1.2, we see

$$
0 \rightarrow H^{k}(B, B-b) \rightarrow H^{k}(M, M-\tilde{b})=\mathbf{Z} \rightarrow H_{b}^{k-n}\left(B ; \mathscr{H}^{n}\right)=0 .
$$

Hence $H^{k}(B, B-b)=\mathbf{Z}$.

Remark. Actually, if $k=n$, the result still holds (see [DW3]). The above method can, with additional care, also prove it.

2.2. Characterization. This subsection shows the equivalence of the conclusion of Theorem 1 (that $B$ is a generalized manifold) with a limitation on the structure of the sheaf. 
Proposition 2.2.1. Let $G$ be a usc decomposition of the $(2 n+1)$-manifold $M$ into shape homology $n$-spheres. Then $M / G$ is a $(n+1)$-gm if, and only if, the set $D \cup K$ is locally finite in $M / G$.

Proof. $(\Rightarrow)$ Suppose $B=M / G$ is a generalized $(n+1)$-manifold. An important property of such spaces is that they are locally orientable, and support Alexander duality. For example, if $U$ is an orientable open set and $A$ an arc in $U$, then

$$
H_{k-1}(U, U-A) \cong H^{1}(A)=0 .
$$

The proof proceeds as follows: we first show $D$ to be countable, then locally finite in $B-K$; then we show that $K$ is locally finite; and, finally, that $E$ is locally finite.

$D$ is countable. Express $D$ as $D_{1} \cup D_{2}$, where $D_{1}$ is countable and $D_{2}$ is dense in itself; we prove that $D_{2}$ is empty. Suppose not. Then $D_{2}$ is a nonempty closed subset of $B-K$ having a dense open continuity set, denoted $C\left(D_{2}\right)$, on which $\mathscr{H}^{n}$ is locally constant (see 1.2.3 and [DyW]). Pick a point $d_{0} \in C\left(D_{2}\right)$ and a neighborhood $U$ of $d_{0}$ in $B$ so that $\widetilde{U}$ is orientable, $U$ is orientable and connected, $U$ is contained in the domain of $\alpha_{d_{0}}$, and $U \cap D_{2} \subset C\left(D_{2}\right)$; select a connected neighborhood $V$ of $d_{0}$ in $U$ so that $\widetilde{V}$ shape deforms in $\widetilde{U}$ to $\tilde{d}_{0}$ and also that $H_{i}(V) \rightarrow H_{i}(U)$ is trivial (where $i=n, n-1$ ); find a neighborhood $W$ of $d_{0}$ in $V$ with the same property with respect to $V$. There is a natural number $\nu$ for all $d \in U \cap C\left(D_{2}\right)$ and $c \in C \cap U$ such that $\alpha_{d}(c)=\nu$.

Fix a point $c_{0}$ in $W \cap C$. Pick two points $d_{1}^{\prime}, d_{2}^{\prime}$ in $W \cap C\left(D_{2}\right)$ distinct from $d_{0}$; find arcs $A_{i}^{\prime}, i=1,2$, in $W-\left(D_{1} \cup\left\{d_{0}\right\}\right)$ so that $\partial A_{i}^{\prime}=\left\{c_{0}, d_{i}^{\prime}\right\}$. Let $A_{i}^{\prime \prime}$ be the path in $A_{i}^{\prime}$ from $c_{0}$ to the first point in $D$ that it meets, say $d_{i}$. Let $A$ be a path in $A_{1}^{\prime \prime} \cup A_{2}^{\prime \prime}$ from $d_{1}$ to $d_{2}$. Note that $A-\partial A \subset C$. By using Lemma 1.3.5 one verifies that $H^{n}(\widetilde{A})=\mathbf{Z}$ and $H^{n+1}(\widetilde{A})=\mathbf{Z}_{\nu}$.

By Lemma 1.5.5, $H_{n}(V, V-A) \cong \mathbf{Z}_{\nu}$. But this is impossible, by Theorem 1.4 .5 , since $B$ is an $(n+1)$-gm. Thus $D$ is countable.

$D$ is discrete. We now show that $D$ is locally finite in $B-K$. Suppose not. Then there is a $d_{0} \in D$ with points $\left\{d_{i} \in D \mid i>0\right\}$ that converge to $d_{0}$. Pick a neighborhood $U$ as before; we assume without loss of generality that each $d_{i}$ lies in $U$. Say $\alpha_{d_{0}}(c)=n_{0}>1$ for all $c$ in $C \cap U$. Noting that $\alpha_{d_{0}}(c)=$ $\alpha_{d_{0}}\left(d_{i}\right) \cdot \alpha_{d_{i}}(c)$ for $c$ close enough to $d_{i}$, we conclude that $\left\{\alpha_{d_{i}}(c) \mid i \geq 0\right\}$ is a bounded set of positive integers. So, there exists a positive integer $l>1$ such that $\alpha_{d_{i}}(c)=l$ for infinitely many of the $i$. Now build an arc $A$ connecting two such points (using that $n>1$ and $B$ is a $(n+1)$-gm), as in the proof of $D$ 's countability, in a small enough neighborhood $V$ of $d_{0}$ to obtain a contradiction.

Thus $D$ is locally finite in $B-K$. 
$K$ is discrete. Let $k_{0}$ be an element of $K$; we show the existence of a neighborhood of $k_{0}$ in $B$ which meets $D \cup K$ in $k_{0}$ only. Let $U, V, W$ be neighborhoods of $k_{0}$ with the usual properties. Suppose there are infinitely many points of $K$ in $W$. Find an arc $A$ in $W-\left\{k_{0}\right\}$ so that $\partial A=A \cap(K \cup D)=$ $A \cap K=\left\{k_{1}, k_{2}\right\}$. By Lemma 1.3.6, $H^{n+1}(\widetilde{A}) \cong \mathbf{Z}$. Alexander duality yields

$$
H_{n}(\tilde{N}, \tilde{N}-\tilde{A}) \cong \mathbf{Z}
$$

where $N$ is $U, V$, or $W$.

Applying Lemma 1.5.5, we have $H_{n}(V, V-A) \cong \mathbf{Z}$. But this is impossible, by Theorem 1.4 .5 , since $B$ is an $(n+1)$-gm. Thus $K$ is discrete.

$E$ is discrete. Thus, without loss of generality, $U \cap K=\left\{k_{0}\right\}$. Note that $H_{k_{0}}^{0}\left(U ; \mathscr{H}^{n}\right) \cong \mathbf{Z}$ is generated by the section that is zero everywhere on $U$ except at $k_{0}$ where it takes the value of a generator of the stalk $\mathscr{H}_{k_{0}}^{n}$ : since $k_{0}$ being an isolated point of $K$ in $U$ means that a basic neighborhood of any element of $\mathscr{H}_{k_{0}}^{n}$ meets any other stalk at 0 , if at all. We now have $\operatorname{dim}(K \cup D) \leq$ 0 . So we may further suppose, by Lemma 1.5.3, that $\left.\mathscr{H}^{n}\right|_{U-(D \cup K)}$ is constant. We have

$0 \rightarrow H_{k_{0}}^{0}\left(U ; \mathscr{H}^{n}\right) \rightarrow H^{n+1}\left(U, U-k_{0}\right) \rightarrow H^{n+1}\left(\widetilde{U}, \widetilde{U}-\tilde{k}_{0}\right) \rightarrow H_{k_{0}}^{1}\left(U ; \mathscr{H}^{n}\right) \rightarrow 0$.

Note that the first three terms in the sequence are isomorphic to $\mathbf{Z}$. Therefore, $H_{k_{0}}^{1}\left(U ; \mathscr{H}^{n}\right)=\mathbf{Z}$. Restricting to $V$ implies the existence of a nonzero section on $U-k_{0}$ which upon restriction to $V-k_{0}$ does not extend to $V$ and every restriction of a section of $U-k_{0}$ to $V-k_{0}$ is an integer multiple of this section's restriction. In particular, if $D \cap V=\left\{d_{1}, d_{2}, \ldots, d_{i}, \ldots\right\}$, the collection $\left\{\alpha_{d_{i}}\left(c_{i}\right)=p_{i} \mid i>0\right\} \quad\left(c_{i}\right.$ close to $\left.d_{i}\right)$ is bounded, which is a contradiction, unless the set is finite.

Thus, $D \cup K$ is locally finite.

$(\Leftarrow)$ Suppose that $D \cup K$ is locally finite. In order to show that $B$ is a generalized $(n+1)$-manifold it suffices to show that $B$ is $\operatorname{LC}^{n+1}$ at each $x \in$ $D \cup K$ and $H_{i}(B, B-x)$ is the appropriate group for all $x$ and $i$.

Choose $x \in D \cup K$. Choose a small enough connected neighborhood $U$ of $x$ so that $U \cap(D \cup K)=\{x\}$ and $\left.\mathscr{H}^{n}\right|_{U-\{x\}}$ is constant.

Whether $x$ is in $D$ or $K$ we get $H^{0}\left(U ; \mathscr{H}^{n}\right)=\mathbf{Z}$. So, by Proposition 2.1.3, we get $B$ is $\operatorname{clc}_{\mathrm{Z}}^{\infty}$ at $x$. Thus, $B$ is an ANR, by Corollary 1.4.12.

We now calculate $H^{n+1}(B, B-x)=H^{n+1}(U, U-x)$.

Case 1: $x \in D$. We observe that $H_{x}^{0}\left(U ; \mathscr{H}^{n}\right)=0$ for any neighborhood $U$ of $x$.

There is a $\nu \in \mathbf{Z}$ so that for any section over $U-\{x\}$ (and by Lemma 1.5.3 we may assume $\left.\mathscr{H}^{n}\right|_{U-\{x\}}$ to be constant, so nontrivial sections exist), the $\nu$ multiple of it extends to $U$. This implies that $H_{x}^{1}\left(U ; \mathscr{H}^{n}\right)$ is a subgroup of 
$\mathbf{Z}_{\nu}$. In any event, the sequence

$$
H_{x}^{0}\left(U ; \mathscr{H}^{n}\right) \rightarrow H^{n+1}(U, U-x) \rightarrow H^{n+1}(\tilde{U}, \tilde{U}-\tilde{x})=\mathbf{Z} \rightarrow H_{x}^{1}\left(U ; \mathscr{H}^{n}\right)
$$

then reveals that $H^{n+1}(U, U-x)=\mathbf{Z}$.

Case 2: $x \in K$. We analyzed isolated points of $K$ in the first half of this proof. Using that analysis, we obtain $\mathrm{Z}=H_{x}^{0}\left(U ; \mathscr{H}^{n}\right)$. In the sequence above, the last map is onto and the first map is an injection. Hence, $H_{x}^{1}\left(U ; \mathscr{H}^{n}\right)$ is either free cyclic or torsion cyclic or trivial, and $H^{n+1}(U, U-x)$ is either $\mathbf{Z}$ or $\mathbf{Z} \oplus \mathbf{Z}$.

Looking at the long exact sequence for the pair $(U, x)$, we observe that the generating section $\sigma$ of $\left.\mathscr{H}^{n}\right|_{U-\{x\}}$ does not extend to a section of $\left.\mathscr{H}^{n}\right|_{U}$, and, hence, it represents a nontrivial element of $H^{0}\left(U-x ; \mathscr{H}^{n}\right)=\mathbf{Z}$ which is not in the image of $H^{0}\left(U ; \mathscr{H}^{n}\right)$ and, hence, is mapped to a nontrivial element $\tau$ of $H_{x}^{1}\left(U ; \mathscr{H}^{n}\right)$; since any nonzero multiple of $\sigma$ does not extend to $\left.\mathscr{H}^{n}\right|_{U}$, $\tau$ represents a nontorsion element of $H_{x}^{1}\left(U ; \mathscr{C}^{n}\right)$. Thus, $H_{x}^{1}\left(U ; \mathscr{C}^{n}\right)$ is free cyclic; this fact, along with the discussion in the previous paragraph, allows us to conclude that $H^{n+1}(U, U-x)=\mathbf{Z}$. This concludes the proof of the proposition.

Remark. It is not assumed in this section that $E$ does not locally separate $B$.

2.3. Proof of Theorem 1. In this subsection we prove Theorem 1, by showing $E=D \cup K$ to be locally finite. The strategy is identical with that used in the last subsection when proving the necessity of the local finiteness of $D \cup K$, except the use there of local duality of a generalized manifold is replaced by a more sophisticated analysis.

Theorem 1. Let $M^{2 n+1}$ be a manifold, $n>1$, and $G$ a uscd of $M$ into compacta having the shape of homology $n$-spheres such that $\operatorname{dim} M / G<\infty$. Suppose the discontinuity set $E=D \cup K$ (see $\S \S 1.2$ and 1.4 ) does not locally separate $B$. Then $M / G$ is a generalized $(n+1)$-manifold. Moreover, $E$ is a locally finite subset of $B$.

Proof. The nondegeneracy set $D$. Since the sheaf $\left.\mathscr{H}^{n}\right|_{B-K}$ is Hausdorff and $B$ is locally connected, we may conclude that $B-K$ is clc by Corollary 2.1.5 (for, given a section on a connected neighborhood of a point of $B$, any section defined on that same neighborhood agreeing with the given section at a point agrees with it everywhere (see section $\S 2.2)$ ). Thus, $B-K$ is an ANR, by 1.4.12.

Given a point $d$ in $D$, we observe that $H_{d}^{0}\left(U ; \mathscr{H}^{n}\right)=0$, for any neighborhood $U$ of $d$. Any section which is nonzero at $d$ is also nonzero on a small neighborhood of $d$; thus, the Leray spectral sequence yields

$$
0=H_{d}^{0}\left(U ; \mathscr{H}^{n}\right) \rightarrow H^{n+1}(U, U-d) \rightarrow H^{n+1}(\tilde{U}, \widetilde{U}-\tilde{d})=\mathbf{Z} \rightarrow \cdots .
$$

Hence,

$$
H^{n+1}(U, U-d)=H^{n+1}(B, B-d)
$$


is either trivial or $\mathbf{Z}$. The sequence also yields $H^{i}(B, B-d)=0$ for $i<n+1$. In consequence, we need only analyze the former case.

Again we describe $D$ as $D_{1} \cup D_{2}$, where $D_{1}$ is countable and $D_{2}$ is dense in itself. The first step is to show that $D_{2}$ is empty.

The critical observation about points in $D$ is

Lemma 2.3.1. Suppose $d_{0} \in D$ and $q \in \mathbf{Z}_{+}(q>1)$. Choose connected neighborhoods $U, V, W$ of $d_{0}$ so that

(1) $W \subset V \subset U$,

(2) $\widetilde{U}$ is orientable,

(3) $H_{i}(W) \rightarrow H_{i}(V)$ has trivial image for $i=n-2, n-1$,

(4) $\widetilde{V}$ (resp. $\widetilde{W}$ ) shape deformation retracts to $\tilde{d}_{0}$ in $\widetilde{U}$ (resp. $\widetilde{V}$ ), and

(5) $U \cap K=\varnothing$.

Then the set

$$
F(q)=\left\{x \in(D \cap W)-\left\{d_{0}\right\}\left|\alpha_{x}\right|_{C}=q \text { and } H_{n}(B, B-x)=0\right\}
$$

consists of at most one point.

Proof. If there are distinct points $x_{1}, x_{2} \in F(q)$, use, among other things, the assumption that $D$ does not locally separate $B$ to build an arc $A$ in $W$ whose endpoints are $x_{1}, x_{2}$ and whose interior lies in $C$. By Lemma 1.3.5, we obtain

$$
\mathbf{Z}_{q} \cong H^{n+1}(\tilde{A}) \cong H_{n}(\tilde{U}, \tilde{U}-\tilde{A}),
$$

the latter map given by Alexander duality.

Since $G$ is $(n-1)$-acyclic, we can apply Lemma 1.5.5 in order to conclude that $H_{n-1}(V, V-A) \cong \mathbf{Z}_{q}$.

We now obtain a contradiction. Let $A_{1}, A_{2}$ denote subarcs of $A$ whose union is $A$. Let $c$ denote their common endpoint. Applying the Mayer-Vietoris Theorem to the triple $\left(V, V-A_{1}, V-A_{2}\right)$ we obtain the following exact sequence:

$$
\begin{array}{clc}
H_{n}\left(V, V-A_{1}\right) \oplus H_{n}\left(V, V-A_{2}\right) & = & H_{n}\left(V, V-x_{1}\right) \oplus H_{n}\left(V, V-x_{2}\right)=0 \\
H_{n}(V, V-c) & = & \mathbf{Z} \\
\downarrow & & \mathbf{Z}_{q}
\end{array}
$$

To show the horizontal map at the beginning of this diagram is an isomorphism, use the fact that $C$ is an $(n+1)$-gm (the technique can be found in [D3, p.192; DyW]).

The exactness of the sequence is patently impossible.

We now prove that $D_{2}$ is void. Choose neighborhoods of $d_{0} \in D_{2}$ as in the above lemma so that $\left.\mathscr{H}^{n}\right|_{U \cap D_{2}}$ is constant. By hypothesis, the intersection of $C$ with each connected open set is a connected set. Let $N=\alpha_{d_{0}}(c)$, where $c$ is any point in $C$. Then $N$ is a bound such that $F(q)=\varnothing$ for all $q>N$. Hence, 
as a consequence of Lemma 2.3.1, we may assume that $H_{n+1}(B, B-x)=\mathbf{Z}$ for all $x \in V-\left\{d_{0}\right\}$. Thus, by Proposition 2.2.1, $D \cap\left(V-\left\{d_{0}\right\}\right)$ is a discrete subset of $V-\left\{d_{0}\right\}$. But this contradicts that $D_{2}$ is dense in itself. Therefore, $D_{2}=\varnothing$. Thus, $D$ is countable.

$D$ is locally finite in $B-K$. Suppose $d_{0} \in D$ is a limit point of $D$. Choose neighborhoods of $d_{0}$ satisfying the hypotheses of Lemma 2.3.1, and so that $\alpha_{d_{0}}$ is defined on $U$. Since $D$ is countable we may write

$$
\left(U-d_{0}\right) \cap D=\left\{d_{1}, d_{2}, d_{3}, \ldots\right\} \text {. }
$$

Then the values of each $\alpha_{d_{i}}$ on $C$ is bounded by the value of $\alpha_{d_{0}}$ on $C$. Hence, we may assume, by Lemma 2.3.1, that

$$
H_{n+1}\left(B, B-d_{i}\right)=\mathbf{Z}
$$

for all $i>0$ (in particular, by 2.2.1, each $d_{i}$ is isolated). So $H_{n+1}\left(B, B-d_{0}\right) \neq$ $\mathbf{Z}$, as another consequence of 2.2.1, and which implies $H_{n+1}\left(B, B-d_{0}\right)=0$. By Lemma 1.5.3, we may assume $\left.\mathscr{H}^{n}\right|_{V-\cup\left\{d_{i} \mid i \geq 0\right\}}$ is constant.

Since $H_{n+1}\left(B, B-d_{0}\right)=0$, excision yields $H_{n+1}\left(V, V-d_{0}\right)=0$. Thus, by the Universal Coefficient Theorem, $H^{n+1}\left(V, V-d_{0}\right)$ is torsion. The long exact sequence of the map restricted to $V$ insures that

$$
\mathbf{Z} \cong H^{n+1}\left(\widetilde{V}, \widetilde{V}-\tilde{d}_{0}\right) \cong H_{d_{0}}^{1}\left(V ; \mathscr{H}^{n}\right) ;
$$

also we may assume that $V$ was chosen so that the map

$$
H^{1}\left(U ; \mathscr{H}^{n}\right) \rightarrow H^{1}\left(V ; \mathscr{H}^{n}\right)
$$

has trivial image, by Proposition 2.1.3 and the fact that $B$ is $(n+1)$-clc. Now consider the following diagram:

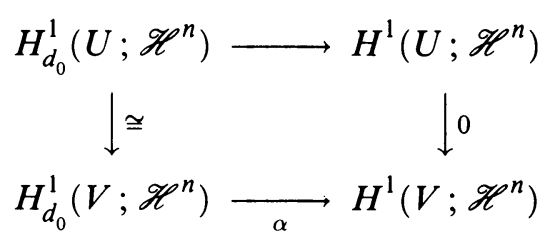

The horizontal maps are from the long exact sequence of the pair $\left(U, U-\left\{d_{0}\right\}\right)$, $\left(V, V-\left\{d_{0}\right\}\right)$. The vertical maps are induced by inclusion. The first vertical map is an excision isomorphism. Thus, the map $\alpha$ has trivial image. Finally, consider the following portion of the long exact sequence for $\left(V, V-\left\{d_{0}\right\}\right)$ :

$$
H^{0}\left(V ; \mathscr{H}^{n}\right) \rightarrow H^{0}\left(V-d_{0} ; \mathscr{H}^{n}\right) \rightarrow H_{d_{0}}^{1}\left(V ; \mathscr{H}^{n}\right) \cong \mathbf{Z} \rightarrow 0 .
$$

The last map, to 0 , is of course $\alpha$. The second map thus being onto implies the existence of an element (i.e., section) of $H^{0}\left(V-d_{0} ; \mathscr{H}^{n}\right)$ which maps onto the generator of $H_{d_{0}}^{1}\left(V ; \mathscr{H}^{n}\right)$. It is clear that no integral multiple of this section extends to $V$, for then that multiple would be in the image of the first of these 
maps, which is identical with the kernel of the second map. Let us call this particular section $\sigma$.

Suppose that, for $c \in C \cap V, \alpha_{d_{0}}(c)=q_{0}$. Now, if $\sigma(c)=l$ (which, without loss of generality, is positive), then consider the function $\sigma^{\prime}: V \rightarrow \mathscr{H}^{n}$ defined by

$$
\sigma^{\prime}(x)= \begin{cases}q_{0} \cdot \sigma, & x \in V-\left\{d_{0}\right\}, \\ l, & x=d_{0} .\end{cases}
$$

We show that $\sigma^{\prime}$ is continuous at $d_{0}$, i.e., a section, contradicting the nonextendibility of $\sigma$.

Consider a sequence of points $x_{i} \rightarrow d_{0}$. We show $\sigma^{\prime}\left(x_{i}\right) \rightarrow \sigma^{\prime}\left(d_{0}\right)$.

Case 1: $\left\{x_{i}\right\} \subset C$. The function is defined so that this case is clear.

Case 2: $\left\{x_{i}\right\} \subset D$. Say $x_{i}=d_{j_{i}}$ and $\alpha_{d_{j_{i}}}(c)=q_{i}$ for $c \in C$ sufficiently close to $d_{j_{i}}$. Note that $q_{0}=\alpha_{d_{0}}\left(d_{j_{i}}\right) \cdot q_{i}$. Then

$$
\sigma^{\prime}\left(x_{i}\right)=q_{0} \cdot l / q_{i}=l \cdot \alpha_{d_{0}}\left(d_{i}\right)
$$

These points in the stalk obviously lie in a neighborhood of $\sigma^{\prime}\left(d_{0}\right)=l$. So, a multiple of $\sigma$ extends to $V$.

This contradiction concludes the proof that $D$ is a discrete subset of $B-K$.

The degeneracy set $K$. We move on to analyze the situation at the points in $K$, a decidedly more subtle matter.

To give the argument a more coherent logical structure we proceed as follows:

(1) show that the isolated points of $K$ are not in the closure of $D$ and, consequently, that $B$ is an $(n+1)$-gm at such points, and

(2) show that $K$ consists entirely of isolated points.

Isolated points of $K$ are isolated points of $D \cup K$. Suppose that $k$ is an isolated point of $K$ which is in the closure of $D$. Choose neighborhoods $U, V$ of $k$ so that $\tilde{U}$ is orientable and retracts to $\tilde{k}, U \cap K=\{k\}, V \subset U$ with $\widetilde{V}$ deforming in $\widetilde{U}$ to $\tilde{k}$, and $U \cap\left(D-\mathrm{cl}_{B} V\right)$ has finite cardinality.

$B$ is $\operatorname{clc}_{\mathrm{Z}}^{n+1}$ at $k$, as can be checked by noting that the only nonzero sections defined on $U$ (or $V$ ) are those which are zero in every stalk except the one over $k$; then apply 2.1.3. By 1.4.12, $U$ is an ANR.

It is clear, by examining sections, that $H^{0}\left(U ; \mathscr{H}^{n}\right)=\mathbf{Z}$. Note that $H_{k}^{1}\left(U ; \mathscr{H}^{n}\right)$ will be trivial (since otherwise there is a nonzero section on $U-k$ with some multiple extending to $U$ ). By Proposition 2.2.1, B fails to be a generalized manifold at $k$. From the exact sequence (Lemma 2.1.2, $A=k$ )

$$
\begin{aligned}
\mathbf{Z} & =H_{k}^{0}\left(U ; \mathscr{H}^{n}\right) \rightarrow H^{n+1}(U, U-k) \rightarrow H^{n+1}(\tilde{U}, \tilde{U}-\tilde{k}) \\
& =\mathbf{Z} \rightarrow H_{k}^{1}\left(U ; \mathscr{H}^{n}\right)=0
\end{aligned}
$$

we must have $H^{n+1}(U, U-k)=\mathbf{Z} \oplus \mathbf{Z}$.

Find an arc $A \subset V$ so that $D \cap V=D \cap A$ and $k \in \partial A$. 
By using the Universal Coefficient Theorem for singular cohomology and Alexander duality we get

$$
\begin{aligned}
H^{n+1}(\widetilde{U}, \widetilde{U}-\widetilde{A}) & =\operatorname{Hom}_{\mathbf{Z}}\left(H_{n+1}(\widetilde{U}, \widetilde{U}-\widetilde{A}), \mathbf{Z}\right) \oplus \operatorname{Ext}\left(H_{n}(\widetilde{U}, \widetilde{U}-\widetilde{A}), \mathbf{Z}\right) \\
& =\operatorname{Hom}_{\mathbf{Z}}\left(H^{n}(\widetilde{A}), \mathbf{Z}\right) \oplus \operatorname{Ext}\left(H^{n+1}(\widetilde{A}), \mathbf{Z}\right) \\
& =\mathbf{Z} \oplus \operatorname{Ext}\left(H^{n+1}(\widetilde{A}), \mathbf{Z}\right)=\mathbf{Z} \oplus \Xi
\end{aligned}
$$

where $\Xi=\operatorname{Ext}\left(H^{n+1}(\tilde{A}), \mathbf{Z}\right)$. Also

$$
H^{n+1}(U, U-A) \cong H^{n+1}(U, U-k)=\mathbf{Z} \oplus \mathbf{Z} .
$$

By applying Lemma 1.5.3, we may assume $\mathscr{H}^{n}$ is constant on $U-(D \cup\{k\})$; thus, there is a generating section of $U-A$, say $\sigma$, with no nonzero multiple of its restriction to $V-A$ extending continuously to $V$, where $V$ is a small neighborhood of $k$ in $U$. We also observe that

$$
H^{0}\left(U-A ; \mathscr{H}^{n}\right) \rightarrow H^{1}\left(U, U-A ; \mathscr{H}^{n}\right) \rightarrow H^{1}\left(U ; \mathscr{H}^{n}\right)
$$

is exact; the last module in this sequence, when restricted to $V$, is trivial. From this we conclude that $H^{1}\left(U, U-A ; \mathscr{H}^{n}\right)=\mathbf{Z}$.

From all the above, and the relative long exact sequence of the map (Lemma 2.1.2), we obtain the exact sequence

$$
\mathbf{Z} \rightarrow \mathbf{Z} \oplus \mathbf{Z} \rightarrow \mathbf{Z} \oplus \mathbf{\Xi} \rightarrow \mathbf{Z} \rightarrow 0 .
$$

Note first that $\Xi$ is finitely generated. Also $\Xi$ is nontrivial; otherwise the last of these maps would be an isomorphism, which in turn would imply that the first is onto-a contradiction.

We now analyze $\Xi$ more carefully in order to conclude that it actually is countably infinitely generated. First a short digression. Let $p_{i}=\alpha_{d_{i}}(c)$ for $c \in C$ close to $d_{i}$. If $\operatorname{gcd}\left(p_{i}, p_{j}\right)=q>1$, then suppose $\pi$ is a path in $V-\left\{k, d_{l} \mid l \neq i, j\right\}$ connecting $d_{i}$ and $d_{j}$. Then Lemma 1.3.5 applies so we know $\breve{H}^{n+1}(\tilde{\pi}) \cong \mathbf{Z}_{q}$.

Proceeding with our analysis of $\Xi$, let $A_{0}=A, c_{i}$ a point in $C$ separating $d_{i}$ and $d_{i+1}$ in $A$, and $A_{i}$ the subinterval of $A$ with endpoints $c_{i}$ and $k$. A Mayer-Vietoris argument in conjunction with the above digression reveals that

$$
H^{n+1}\left(\tilde{A}_{i}\right) \rightarrow H^{n+1}\left(\tilde{A}_{i+1}\right)
$$

is onto with kernel isomorphic to $\mathbf{Z}_{p_{i}}$. Also, $H^{n+1}\left(\cap \tilde{A}_{i}\right)=H^{n+1}(\tilde{k})=0$.

Let $\Gamma=H^{n+1}(\tilde{A})$. The above shows we can apply Lemma 1.5.1 to $\Gamma$, yielding

$$
\Gamma \cong \sum \Gamma(p),
$$

where the sum is taken over all primes $p$, and either $\Gamma(p) \neq 0$ for infinitely many $p$ or, for some prime $p, \Gamma(p)$ is (countably) infinitely generated. Also, $\Xi=\operatorname{Ext}(\Gamma, \mathbf{Z}) \cong \Pi \Gamma(p)$, by a well-known property of Ext (see [R, p. 199]). In particular, $\Xi$ cannot possibly be finitely generated. 
This contradiction proves the assertion that the isolated points of $K$ are isolated points of $K \cup D$.

$K$ is discrete. Let $K^{\prime}$ denote the points of $K$ which are not isolated points of $K$. Note that $K^{\prime}$ is a closed subset of $B$. We conclude the proof of Theorem 1 by showing that $K^{\prime}$ is void. We define $C\left(K^{\prime}\right)$ to be an open dense subset of $K^{\prime}$ where the sheaf $\left.\mathscr{H}^{n}\right|_{K^{\prime}}$ is locally constant (see 1.2.3 and [DyW]). The strategy is to first show that every point of $C\left(K^{\prime}\right)$ is a limit point of $K-K^{\prime}$ (which is obvious for points of $K^{\prime}$ isolated in $\left.K^{\prime}\right)$; we then establish a contradiction using this fact. Since $C\left(K^{\prime}\right)$ is an open dense subset of $K^{\prime}$, we conclude that $C\left(K^{\prime}\right)=K^{\prime}=\varnothing$.

Let $k \in C\left(K^{\prime}\right)$. Let $U$ be a neighborhood of $k$ in $B$ so that $\alpha_{k} \mid U \cap K^{\prime}$ is continuous with $\tilde{U}$ orientable, and $V \subset U$ a neighborhood such that $\widetilde{V}$ [shape] deforms to $\tilde{k}$ in $\widetilde{U}$.

Suppose that $k$ is not isolated in $K^{\prime}$. So there are infinitely many points of $K^{\prime}$ in any neighborhood of $k$. Furthermore, suppose that $k$ is not in the closure of $K-K^{\prime}$. Then we can assume that $U$ contains no isolated points of $K$. Note that $H^{n+1}\left(U, U-\left\{k^{\prime}\right\}\right)$ is either trivial or $\mathbf{Z}$ for every $k^{\prime} \in U \cap K^{\prime}$, since $H_{x}^{0}\left(U ; \mathscr{H}^{n}\right)=0$.

Claim 1. There cannot be distinct points $k_{1}, k_{2} \in V \cap K^{\prime}$ with

$$
H^{n+1}\left(V, V-\left\{k_{i}\right\}\right)=0 \text { for } i=1,2 \text {. }
$$

Else, using the long exact sequence of the map as was done previously, we detect sections

$$
\sigma_{i}: U-\left\{k_{i}\right\} \rightarrow \mathscr{H}^{n}
$$

so that no (nonzero) multiple of $\sigma_{i}$ extends to $U, i=1,2$. Because $k$ is not in the closure of $K-K^{\prime}$ we have $\sigma_{i} \mid U-K^{\prime} \equiv 0$ for each $i$. Moreover, since $U \cap K^{\prime}$ is in the continuity set of $K^{\prime}$, we have $\sigma_{i}(x)=m_{i}>0$ for all $x \in U \cap K^{\prime}$. But then $m_{2} \cdot \sigma_{1}$ can be extended continuously to $U$ by comparing it to $m_{1} \cdot \sigma_{2}$ which is defined at $k_{1}$. Claim 1 is verified.

Before proceeding, we should observe that $U$ is an ANR. This is suspect only at points $k^{\prime} \in U \cap K=U \cap C\left(K^{\prime}\right)$, a suspicion which the following claim allays.

Claim 2. The image of $h: H^{0}\left(U ; \mathscr{H}^{n}\right) \rightarrow H^{0}\left(V^{\prime} ; \mathscr{H}^{n}\right)$ is finitely generated (where $V^{\prime}$ is a sufficiently small neighborhood of $k^{\prime}$ ). Pick distinct nonzero elements $\sigma_{1}, \sigma_{2}$ of this image, with $\tilde{\sigma}_{1}, \tilde{\sigma}_{2}$ respective elements of $h^{-1}\left(\sigma_{1}\right)$, $h^{-1}\left(\sigma_{1}\right)$. Let $s_{i}=\sigma_{i}\left(k^{\prime}\right), i=1,2$. Note that $s_{i} \neq 0$; otherwise $\sigma_{i}$ will be the zero section since $V^{\prime}$ was chosen small, $k^{\prime} \in C\left(K^{\prime}\right)$, and $U \cap\left(K-K^{\prime}\right)=\varnothing$. Let $t$ be the greatest common divisor of $s_{1}$ and $s_{2}$. By taking the appropriate linear sum of $\sigma_{1}$ and $\sigma_{2}$, we can obtain a nontrivial section $\sigma_{3}$ of $V^{\prime}$ which is the restriction of a section $\tilde{\sigma}_{3}$ of $U$ such that $\sigma_{3}\left(k^{\prime}\right)=t$. Let $t_{0}$ be the minimum $t$ taken over all possible pairs of such sections, and let $\sigma$ be the corresponding section over $V^{\prime}$. 
Then $\sigma$ generates the image of $h$. If $\tau$ is a nonzero element of the image of $h$, then $\operatorname{gcd}\left(\sigma\left(k^{\prime}\right), \tau\left(k^{\prime}\right)\right)=t_{0}$, otherwise the minimality of $t_{0}$ is contradicted. Thus, the image is either trivial or infinite cyclic, proving Claim 2.

Therefore, $U$ is $(n+1)-\mathrm{clc}_{\mathrm{z}}$ and, so, by $2.1 .3, \mathrm{clc}_{\mathbf{z}}^{\infty}$. Hence, $U$ is an ANR, by 1.4 .12 .

It must be that, for the given $k, H^{n+1}(U, U-\{k\})=0$, for otherwise $U$ is a neighborhood of $k$ which is an $(n+1)$-gm (by Claim 1$)$ that has infinitely many sheaf discontinuities, contradicting Proposition 2.2.1. As a consequence, there are no points in $\left(U \cap K^{\prime}\right)-\{k\}$ since they too would have such neighborhoods. Thus $k$ is isolated in $K^{\prime}$. But $k \in K^{\prime}$, not being in the closure of $K-K^{\prime}$, must then be isolated in $K$-contradicting the defining property of points in $K^{\prime}$.

So we assume that each $k \in C\left(K^{\prime}\right)$ which is not isolated in $K^{\prime}$ is a limit point of $K-K^{\prime}$. This is necessarily true of any isolated point of $K^{\prime}$.

Note that $U-K^{\prime}$ is an $(n+1)$-gm (but now $U$ itself is not necessarily an ANR!). Thus, for any arc $A$ in $U-K^{\prime}, H^{n}(U, U-A)=0$ (this can be seen by first excising $K^{\prime}$ to form the pair $\left(U-K^{\prime}, U-\left(K^{\prime} \cup A\right)\right.$ ) and again using the technique appearing in [D3, p. 192]). The Universal Coefficient Theorem implies that $H_{n}(U, U-A)$ is a torsion module.

Find an arc $A^{\prime}$ in $V-K^{\prime}$ with $\left(A^{\prime}-\partial A^{\prime}\right) \subset C$ and $\partial A^{\prime}=\left\{k_{1}, k_{2}\right\} \subset K-K^{\prime}$.

Let $c \in A^{\prime}-\left\{k_{1}, k_{2}\right\}$ and let $A_{i}$ denote the closure of the component of $A^{\prime}-\{c\}$ containing $k_{i}$. Consider the sequence

$$
H^{0}\left(A_{i} ; \mathscr{H}^{n}\right) \rightarrow H^{0}\left(k_{i} ; \mathscr{H}^{n}\right) \rightarrow H^{1}\left(A_{i}, k_{i} ; \mathscr{H}^{n}\right) \rightarrow H^{1}\left(A_{i} ; \mathscr{H}^{n}\right) \rightarrow 0 .
$$

The first map is a natural isomorphism in this case since the generating sections are equivalent. Thus, the third map is an isomorphism.

It can be calculated, using Proposition 12.2 of [ $\mathrm{Br} 1$, p. 59] and our explicit knowledge of the structure of $\left.\mathscr{C}^{n}\right|_{A_{i}}$, that $H^{1}\left(A_{i}, k_{i} ; \mathscr{C}^{n}\right)$ is isomorphic to $H^{1}\left(A_{i} ; \mathbf{Z}_{A_{i}-k_{i}}\right) \cong 0$. So, $H^{1}\left(A_{i} ; \mathscr{H}^{n}\right)=0$.

Now we examine the Mayer-Vietoris sequence

$$
\cdots \rightarrow H^{0}\left(A_{1} ; \mathscr{H}^{n}\right) \oplus H^{0}\left(A_{2} ; \mathscr{H}^{n}\right) \rightarrow H^{0}\left(c ; \mathscr{H}^{n}\right) \rightarrow H^{1}\left(A^{\prime} ; \mathscr{H}^{n}\right) \rightarrow 0 .
$$

The first map is trivial because the sections defined over the $A_{i}$ 's are trivial over $c$. Thus the second map is an isomorphism; $H^{0}\left(c ; \mathscr{H}^{n}\right)$ being infinite cyclic abelian implies that $H^{1}\left(A^{\prime} ; \mathscr{H}^{n}\right)$ is as well.

Choose $k_{3} \in(K \cap V)-\left\{k_{1}, k_{2}\right\}$ and an arc $A_{3}$ from $c$ to $k_{3}$ in $V$ so that $A_{3} \cap A^{\prime}=\{c\}$ and $A_{3} \cap K=\left\{k_{3}\right\}$. By applying the Mayer-Vietoris argument to the pair $\left(A^{\prime}, A_{3}\right)$, we find that

$$
H^{1}\left(A^{\prime} \cup A_{3} ; \mathscr{H}^{n}\right)=\mathbf{Z} \oplus \mathbf{Z}
$$

Let $A=A^{\prime} \cup A_{3}$. First we get, by Alexander duality,

$$
J=\operatorname{im}\left\{H_{n-1}(\tilde{V}-\tilde{A}) \rightarrow H_{n-1}(\tilde{U}-\tilde{A})\right\}
$$


is $\mathbf{Z} \oplus \mathbf{Z}$ (we know the map $H_{n}(\tilde{U}-\widetilde{A}) \rightarrow H_{n}(\tilde{U})$ is onto, because $\tilde{k}_{0} \subset(\tilde{U}-\widetilde{A})$ is the essential carrier of the latter group).

Using the Vietoris-Begle Theorem, we conclude that

$$
H_{n-1}(V-A) \rightarrow H_{n-1}(U-A)
$$

has image isomorphic to $J$.

Finally, we consider

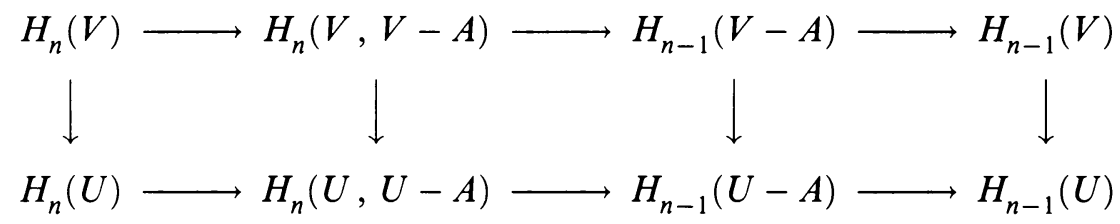

The second vertical arrow is an excision isomorphism of trivial groups, the fourth is a trivial map. But then we must have $J=0$, a contradiction.

Thus, $C\left(K^{\prime}\right)$ is empty. In consequence, $K^{\prime}$ is empty, implying $K$ is a discrete subset of $B$. This concludes the proof of Theorem 1 .

2.4. Corollaries, examples, and questions. This section shows the possible types of sheaf structures that can be attained, and a certain limitation is proved in case the source space is the $(2 n+1)$-sphere.

Proposition 2.4.1. Suppose $G$ to be a decomposition of a $(2 n+1)$-manifold $M$ into continua having the shape of homology $n$-spheres with $\operatorname{dim} M / G<\infty$ such that $E=D \cup K$ does not locally separate $M / G$. Suppose $H^{n}(M)=0$. If $M / G$ is orientable (e.g., if it is simply connected), then $K$ consists of at most one point.

Proof. The long exact sequence associated with the map yields

$$
0 \rightarrow \check{H}^{0}\left(M / G ; \mathscr{H}^{n}\right) \rightarrow H^{n+1}(M / G)=\mathbf{Z} .
$$

Since each point of $K$ corresponds to a generator of $\check{H}^{0}\left(M / G ; \mathscr{H}^{n}\right)$ due to local finiteness of $K$, the conclusion follows.

The following is an easy consequence of the fact that monotone maps induce epimorphisms of the fundamental group.

Corollary 2.4.2. Each decomposition of the $(2 n+1)$-sphere into homology $n$ spheres (with finite-dimensional image and exceptional set $E$ not locally separating the decomposition space) has at most one degenerate element.

The example of the Introduction illustrates $K$ being nonempty.

Example. Let $\Sigma$ denote the Poincaré homology sphere; there is a 120 -to-1 covering of the 3 -sphere to $\Sigma$. Let $\mathscr{G}$ denote the covering transformations for this cover. This group action extends naturally to $E^{4}$, the open cone on $S^{3}$. Now let this group act diagonally on the product $S^{3} \times E^{4}$; let the orbit space be $M$. There is a natural decomposition of the 7-manifold $M$ into 3-spheres 
and an exceptional homology 3-sphere; the latter is the image, under the orbit map, of $S^{3} \times\{0\}$, whereas the former are the images, under the orbit map, of $\mathscr{G}(x) \times r \cdot S^{3}$, where $\mathscr{G}(x)$ denotes the orbit of the given point $x \in S^{3}$ and $r \cdot S^{3}$ denotes the sphere in $E^{4}$ of radius $r$ centered at the origin.

Note that $K=\varnothing$ and $D$ is a singleton whose winding function equals 120 on its continuity set.

For nondegenerate decompositions of $S^{2 k+1}$ into copies of $S^{k}$ ( $k$ odd), we refer the reader to [CD2]. They also show that for even $k$ no such decompositions exist; this is achieved by computing the Hopf invariant (which is 0 for even $k$ ) to be an upper bound on the cardinality of $D$. A question we have left unanswered: Is there an analogous bound for a nondegenerate homology $n$-sphere decomposition of $S^{2 n+1}$ ?

Corollary 2.4.3. There is no uscd of Euclidean $(2 n+1)$-space into (homology) n-spheres.

Proof. We consider the Leray spectral sequence of the decomposition map $p$ with the families of compact supports $c, \phi$ on domain and image. Clearly, $\breve{H}_{\phi}^{0}\left(B ; \mathscr{H}^{n}\right)=0$ and $H_{c}^{n+1}\left(E^{2 n+1}\right)=0$. Thus, $H_{c}^{n+1}(B)=0$.

On the other hand, since $B$ is an orientable $(n+1)$-gm, duality theory tells us that $H_{c}^{n+1}(B) \cong H_{0}(B) \cong \mathbf{Z}$, a contradiction.

Questions. (1) Is there a nondegenerate homology $n$-sphere decomposition of $S^{2 n+1}$ each of whose elements is a nontrivial homology $n$-sphere? Are there any such decompositions for even $n$ ? (If all the elements are sphere-like and $B$ is a manifold, this is known to be impossible.)

(2) Given any nondegenerate homology $n$-sphere decomposition of $S^{2 n+1}$, is there an invariant which measures the maximum allowable number of points in $D$ ? If so, for which $n$ can this maximum be realized?

(3) For which $n$ and $k, n>k>0$ (if any), is there a homology $n$-sphere decomposition of $S^{2 n+1-k} \times S^{k}$ ?

\section{A CLASS OF PARTIALLY ACYCLIC MANIFOLD DECOMPOSITIONS}

In this section, we prove Theorem 2 of the Introduction. The section is organized as follows: $\S 3.1$ is a discussion (under more general hypotheses than those of Theorem 2) of pertinent quantities in the Leray spectral sequence and the immediately discernable connectivity properties of $B ; \S 3.2$ shows the equivalence of $B$ being a $k$-gm over $\mathbf{Z}$ with local finiteness of the discontinuity set; $\S 3.3$ demonstrates that the decompositions of Theorem 2 must always have locally finite discontinuity set; and $\$ 3.4$ contains some examples and corollaries.

It should be noted that there is a rather slight difference in some of the details here from those of $\S 2$. Here, the sheaf $\mathscr{C}^{k-1}$ appears to play a role, though for the most part we avoid dealing with it by deepening our understanding of how to calculate Čech cohomology with coefficients in $\mathscr{H}^{n}$ of sufficiently high dimensions. 
3.1. The Leray spectral sequence of the decomposition map. In this subsection, we calculate the crucial parts of the Leray spectral sequence of the decomposition map.

Assume that $G$ is a uscd of the orientable $(n+k)$-manifold into subcontinua so that the following properties are satisfied:

(†) For all $g \in G, \widetilde{H}_{i}(g)=0$ for $i \in\{0,1, \ldots, k-2\}$.

(††) Each $g \in G$ has the shape of a closed orientable $n$-manifold.

$(\dagger \dagger \dagger) \operatorname{dim} M / G<\infty$.

Note that $E_{2}^{p, q}=0$ for all $p>k$, since $\operatorname{dim} M / G=k$, by $(\dagger \dagger \dagger)$, and by the (sheaf theoretic) fact that $H^{i}(M / G ; \mathscr{S})=0$ for all $i>k$; for all $q>n$, by $(\dagger \dagger)$; and, if $0 \leq p \leq k$, for $0<q<k-1, n-k+1<q<n$, by ( $\dagger$ ). In particular, when $E_{\infty}$ is calculated, we obtain the following data:

(1) $E_{\infty}^{p, 0}=E_{2}^{p, 0}$ for $0 \leq p<k$,

(2) $E_{\infty}^{k, 0}=E_{2}^{k, 0} /$ image $d_{k-1}^{0, k-1}$,

(3) $E_{\infty}^{p, n}=E_{2}^{p, n}$ for $1 \leq p \leq k$,

(4) $E_{\infty}^{0, n}=\operatorname{ker} d_{k-1}^{0, n}$

(5) $E_{\infty}^{0, k-1}=\operatorname{ker} d_{k-1}^{0, k-1}$,

(6) $E_{\infty}^{1, k-1}=E_{2}^{1, k-1}$, and

(7) $E_{\infty}^{0, k}=\operatorname{ker} d_{2}^{0, k}$.

Thus, for supports $\psi, \phi$ on $M, B$, respectively, we obtain

(a) $H_{\phi}^{i}(U) \cong H_{\phi(\psi)}^{i}(\widetilde{U})$, for $i<k-1$,

(b) an exact sequence of homomorphisms

$$
0 \rightarrow H_{\phi}^{k-1}(U) \rightarrow H_{\phi(\psi)}^{k-1}(\tilde{U}) \rightarrow H_{\phi}^{0}\left(U ; \mathscr{H}^{k-1}\right) \rightarrow H_{\phi}^{k}(U) \rightarrow H_{\phi(\psi)}^{k}(\tilde{U}),
$$

and

(c) $H_{\phi}^{k}\left(U ; \mathscr{H}^{n}\right) \cong H_{\phi(\psi)}^{n+k}(\widetilde{U})$.

The information in this list is enough for this investigation.

For example, letting $\psi, \phi$ be the family of closed subsets of $M, B$, we see, by (a), that $B$ is $\operatorname{clc}_{\mathrm{Z}}^{k-2}$ (see the proof of 2.1.3). By (b), $B$ is in fact cohomologically locally connected in all dimensions except possibly $k$.

By using $\phi=\{x\}$ in (a) and (b), we obtain $H^{i}(B, B-x)=0$ for all $x \in B$ and $i<k$.

From (b) and (c), we may derive that $C=\left\{x \in B \mid \mathscr{H}^{n}\right.$ is locally constant at $x\}$ is a $k$-gm (see 2.1.3-2.1.6).

Another consequence of (b) is that $B$ is $k-\operatorname{clc}_{\mathbf{Z}}$ at $x \in B$ if, and only if, $B$ is $0-\operatorname{clc}_{\not P^{k-1}}$ at $x$, an analogue of 2.1.3.

3.2. Characterization. In this subsection we prove the analogue of 2.2.1. The hypotheses on $M$ and $G$ are the same as in $\S 3.1$.

Proposition 3.2.1. Assume $G$ is a uscd of an $(n+k)$-manifold $M$ satisfying the hypothesis of $\S 3$. Then $B$ is a k-gm if, and only if, $E=D \cup K$ is locally finite. 
Proof. $(\Rightarrow)$ This proof is nearly identical to that of Proposition 2.2.1 except, when calculating the homology groups, we replace $n=(2 n+1)-(n+1)$ by $k-1=(n+k)-(n+1)$.

$(\Leftrightarrow) B$ is an $A N R$. $B$ is $\operatorname{clc}_{\mathrm{Z}}^{k-1}$ and $\operatorname{dim} B<\infty$. Therefore, it suffices to show that $B$ is $k$-clc $\mathrm{z}$.

If $\mathscr{H}^{n}$ is Hausdorff on a neighborhood of $x \in B$, then

$$
H^{0}\left(U ; \mathscr{H}^{k-1}\right) \subset H^{k-1}(\tilde{x})
$$

(the latter group is finitely generated) for a small enough connected neighborhood $U$ of $x$. Thus, $B$ is $k-\operatorname{clc}_{\mathrm{z}}$ at $x$.

If $x \in K$, then choose a connected neighborhood $U$ which misses $E-\{x\}$ and so that $\mathscr{H}^{n}$ is constant on $U-x$. Then $U-x$ is connected. Clearly,

$$
H^{0}\left(U ; \mathscr{H}^{k-1}\right) \subset H^{0}\left(U-x ; \mathscr{C}^{k-1}\right) \oplus H_{x}^{0}\left(U ; \mathscr{H}^{k-1}\right) .
$$

Both summands are finitely generated. So $B$ is $k-\operatorname{clc}_{\mathrm{Z}}$ at $x$. Hence, $B$ is $\mathrm{Clc}_{\mathrm{Z}}^{\infty}, \mathrm{LC}^{1}$, and, consequently, an ANR.

$B$ is a cohomology $k$-manifold over $\mathbf{Z}$. By the results of $\S 3.1$, we have $H^{i}(B, B-x)=0$ for all $x \in B$ and $0 \leq i \leq k-1$. Looking at item (4) in the list of $\S 3.1$, we note that

$$
\check{H}_{x}^{k}\left(U ; \mathscr{H}^{n}\right) \cong H^{n+k}(\widetilde{U}, \widetilde{U}-\tilde{x})=\mathbf{Z}
$$

for small neighborhoods $U$ of $x$. So it suffices to demonstrate that

$$
\check{H}_{x}^{k}\left(U ; \mathscr{H}^{n}\right) \cong H_{x}^{k}(U ; \mathbf{Z}),
$$

for a suitably chosen neighborhood $U$. This is trivial for $x \notin E$.

So we assume $x \in E$, and $U$ a connected neighborhood of $x$ such that $U \cap E=\{x\}$ and, by 1.5.3, $\left.\mathscr{C}^{n}\right|_{U-x}$ is constant. Then consider the following diagram

$$
\begin{aligned}
& H^{k-1}(U) \stackrel{\cong}{\longleftarrow} \check{H}^{k-1}\left(U ; \mathscr{H}^{n}\right) \\
& \downarrow \alpha_{1} \quad \downarrow \beta_{1} \\
& H^{k-1}(U-x) \stackrel{\cong}{\longleftarrow} \check{H}^{k-1}\left(U-x ; \mathscr{C}^{n}\right) \\
& \downarrow_{2} \quad \downarrow \beta_{2}
\end{aligned}
$$

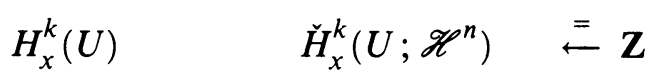

$$
\begin{aligned}
& \downarrow^{\alpha} \quad \downarrow_{3} \\
& H^{k}(U) \stackrel{\cong}{\longleftarrow} \check{H}^{k}\left(U ; \mathscr{H}^{n}\right) \\
& \downarrow^{\alpha} \quad \downarrow \beta_{4} \\
& H^{k}(U-x) \stackrel{\cong}{\longleftarrow} \check{H}^{k}\left(U-x ; \mathscr{H}^{n}\right)
\end{aligned}
$$


The vertical maps come from the appropriate long exact sequences of the pair $(U, U-x)$. The second and fourth horizontal maps are obviously isomorphisms, since $\left.\mathscr{H}^{n}\right|_{U-x}$ is constant. We argue that we can find isomorphisms (represented by the first and third horizontal maps) so that the squares in the diagram commute. Once we have established this a rather routine diagram chase shows that $H^{k}(U, U-x) \cong \mathbf{Z}$.

The key to understanding the indicated isomorphisms is applying dimension theory when calculating $\breve{H}^{i}\left(U ; \mathscr{H}^{n}\right)$. The reader is referred to [S, pp. 327-328], as we content ourselves, for now, with the description

$$
\check{H}^{i}\left(U ; \mathscr{H}^{n}\right)=\underline{\lim }\left\{H^{i}\left(\mathscr{U} ; \mathscr{H}^{n}\right)\right\},
$$

where the limit is being taken over all open covers $\mathscr{U}$ of $U$ (which form an inverse system via refinement). Since $U$ is $k$-dimensional, for every open cover $\mathscr{U}$ of $U$ there is a refinement $\mathscr{V}$ of order $k+1$ such that $x$ is not in the intersection of any two elements of $\mathscr{V}$. A glance at the definition of $H^{i}\left(\mathscr{V} ; \mathscr{H}^{n}\right)$ will show that, because $\left.\mathscr{H}^{n}\right|_{U-x}$ is constant, we can use the constant sheaf $U \times \mathbf{Z}$ rather than $\mathscr{H}^{n}$ for $i>1$. Thus, there is a natural isomorphism, for $i>1$, between the limits

$$
\stackrel{\lim }{\longrightarrow}\left\{H^{i}\left(\mathscr{U} ; \mathscr{H}^{n}\right)\right\} \cong \lim \left\{H^{i}(\mathscr{U} ; \mathbf{Z})\right\} .
$$

In particular, since $k-1>1$, we obtain the desired isomorphisms. Thus, $B$ is a $k$-gm.

3.3. Proof of Theorem 2. In this subsection we prove Theorem 2. In particular, in addition to the hypotheses of the previous subsections of this section, we assume

(1) $\mathscr{H}^{n}$ is a Hausdorff space (i.e., $K=\varnothing$ ), and

(2) $D$ does not locally separate $B$.

Theorem 2. Let $M^{n+k}$ be an orientable manifold, $n \geq k \geq 3$. Let $G$ be a uscd of $M$ into compacta having the shape of connected, closed, orientable $n$ manifolds with $\check{H}_{i}(g) \cong 0$ for $0<i<k-1$. If $\mathscr{H}^{n}[p]$ (see $\left.\S 1.1\right)$ is Hausdorff, $D$ does not locally separate $B$, and $\operatorname{dim} M / G<\infty$, then $M / G$ is a generalized k-manifold.

Proof. Note that $B$ is an ANR by (1), since $\check{H}^{0}\left(U ; \mathscr{C}^{k-1}\right) \subset \check{H}^{k-1}(\tilde{x})$ for any connected open set $U$ and $x \in U$. Moreover, $H^{i}(B, B-x)=0$ for any $x \in B$ and $i \neq k$. Given a connected subset $U$, we have $U \cap C$ connected by (2).

By the proposition of the previous subsection, it suffices to show $D$ to be locally finite in $B$. Let

$$
D_{1}=\{x \in D \mid x \text { is isolated in } D\}
$$

and $D_{2}=D-D_{1}$. Note that $D_{1}$ is an open subset of $D$ and is countable; $D_{2}$ is closed in $B$. We show $D_{2}=\varnothing$. 
Before proceeding, we state the following analogue of Lemma 2.3.1; the proof here has the same strategy, the only significant difference being in indices on the algebraic topological functors.

Lemma 3.3.1. Suppose $d_{0} \in D$ and $q \in \mathbf{Z}_{+}(q>1)$. Choose connected neighborhoods $U, V, W$ of $d_{0}$ so that

(1) $W \subset V \subset U$,

(2) $H_{i}(W) \rightarrow H_{i}(V)$ has trivial image for $i=k, k-1$, and

(3) $\widetilde{V}$ (resp. $\widetilde{W}$ ) shape deformation retracts to $\tilde{d}_{0}$ in $\widetilde{U}$ (resp. $\tilde{V}$ ).

Then the set

$$
F(q)=\left\{x \in(D \cap W)-\left\{d_{0}\right\}\left|\alpha_{x}\right|_{C}=q \text { and } H^{k}(B, B-x)=0\right\}
$$

consists of at most one point.

Proof. If there are distinct points $x_{1}, x_{2} \in F(q)$, build an arc $A$ in $W$ whose endpoints are $x_{1}, x_{2}$ and whose interior lies in $C$, using the hypothesis that $D$ does not locally separate $B$. By Lemma 1.3 .5 , we obtain

$$
\mathbf{Z}_{q} \cong \check{H}^{n+1}(\widetilde{A}) \text {. }
$$

Since $G$ is $(n-1)$-acyclic and $k \leq n, G$ is $(k-1)$-acyclic; thus, we can apply Lemma 1.5.5, which allows us to conclude that $H_{k-1}(V, V-A) \cong \mathbf{Z}_{q}$.

We now obtain a contradiction. Let $A_{1}, A_{2}$ denote subarcs of $A$ whose union is $A$. Let $c$ denote their common endpoint. Applying the Mayer-Vietoris Theorem to the excisive triple $\left(V, V-A_{1}, V-A_{2}\right)$, we obtain the following exact sequence:

$$
\begin{array}{ccc}
H_{k}\left(V, V-A_{1}\right) \oplus H_{k}\left(V, V-A_{2}\right) & = & H_{k}\left(V, V-x_{1}\right) \oplus H_{k}\left(V, V-x_{2}\right)=0 \\
\downarrow & = & \mathbf{Z} \\
H_{k}(V, V-c) & & \\
H_{k-1}(V, V-A) & = & \mathbf{Z}_{q}
\end{array}
$$

To show the horizontal map at the beginning of this diagram is an isomorphism, use the fact that $C$ is a $k$-gm (for details on the technique see [D3, p. 192; DyW]).

The exactness of this sequence is impossible.

Select $d_{0} \in C\left(D_{2}\right)$. Find a connected neighborhood $U$ of $d_{0}$ such that

$$
\left.\mathscr{C}^{n}\right|_{U \cap D_{2}} \cong\left(U \cap D_{2}\right) \times \mathbf{Z} \text {. }
$$

By hypothesis, $C \cap U$ is connected. Let $N=\alpha_{d_{0}}(c)$, where $c$ is any point in $C$. Then $N$ is a bound such that $F(q)=\varnothing$, for all $q>N$. By Lemma 3.3.1, we may assume $U$ to be chosen such that, for each $d \in(D \cap U)-\left\{d_{0}\right\}$, $H^{k}(U, U-d)=\mathbf{Z}$; in other words, $U-\left\{d_{0}\right\}$ is a $k$-gm. We may assume the winding functions have bounded value. 
By Proposition 3.2.1, $D \cap\left(V-\left\{d_{0}\right\}\right)$ is a discrete subset of $V-\left\{d_{0}\right\}$. But this contradicts that $D_{2}$ is dense in itself.

Thus, $C\left(D_{2}\right)=\varnothing=D_{2}$. So $D$ is locally finite, 3.2.1 then implying that $B$ is a $k-\mathrm{gm}_{\mathbf{z}}$.

3.4. Immediate corollaries, examples, and questions. We start out this subsection with some consequences of Theorem 2 .

Theorem 3.4.1. There is no nondegenerate codimension- $k$ manifold decomposition $G$ of $S^{n+k}$ with the following properties:

(1) For all $g \in G, \widetilde{H}_{i}(g)=0$ for $i \in\{0,1,2, \ldots, k-2\}$.

(2) $\operatorname{dim} M / G<\infty$.

(3) $\mathscr{H}^{k-1}$ is constant and the rank of $\mathscr{H}_{x}^{k-1}$ as a Z-module is at least 2 , for some $x \in B$.

(4) The exceptional set $E=D$ does not locally separate $B$.

Addendum. Item (3) can be weakened to: $\left(3^{\prime}\right)$ there is a sheaf embedding of $B \times F$ into $\mathscr{H}^{k-1}$, where $F$ is a free $\mathrm{Z}$-module of rank at least 2 .

Proof. Suppose such a $G$ exists. Property (3) means that $H^{0}\left(B ; \mathscr{H}^{k-1}\right)$ is a free $\mathbf{Z}$-module on at least two generators. On the other hand, from the Leray spectral sequence we get an injection

$$
H^{0}\left(B ; \mathscr{H}^{k-1}\right) \rightarrow H^{k}(B) \text {. }
$$

We further realize that $B$ is a simply-connected compact $k$-gm, hence is orientable; so, by duality, $H^{k}(B) \cong \mathbf{Z}$. This is a contradiction.

Corollary 3.4.2. There is no approximate fibration defined on $S^{n+k}$ with fiber $F$ such that

(1) $F$ has the shape of a closed orientable $n$-manifold.

(2) $\widetilde{H}_{i}(F)=0$. for $i \in\{0,1, \ldots, k-2\}$.

(3) $\operatorname{rank} H_{k-1}(F) \geq 2$.

Proof. For an approximate fibration, $\mathscr{H}^{k-1}$ is locally constant and the set $E$ is empty; by simple connectivity of $B, \mathscr{H}^{k-1}$ is globally constant.

Proposition 3.4.3. There is no uscd $G$ of $S^{n+k}$ satisfying the hypotheses of Theorem 2 such that rank $H_{k-1}(g)=0$ for all $g \in G$.

Addendum. There is no uscd $G$ of a closed manifold $M^{n+k}(k \leq n)$ satisfying the hypotheses of Theorem 2 such that $\operatorname{rank} H_{k-1}(g)=0$ for all $g \in G$, if, in addition, $H_{1}(M)=0$ and $\operatorname{rank} H_{n}(M)=0$.

Proof. If rank $H_{k-1}(g)=0$ for all $g \in G$, then $\mathscr{H}^{k-1}$ is trivial with trivial stalks. On the one hand, since $B$ is a compact simply-connected $k$-gm, we have 
$H^{k}(B) \cong \mathbf{Z}$; on the other, we have, by item (b) in $\S 3.1, H^{k}(B) \subset H^{k}\left(S^{n+k}\right)=0$. This is impossible. Proving the addendum is similar: note that $B$ is a compact, orientable $k$-gm, since $H_{1}(M)=0$; and that $H^{k}\left(M^{n+k}\right)=0$ because of the constraint on the rank of $H_{n}(M)$.

Theorem 3.4.4. There is no uscd $G$ of $E^{n+k}$ satisfying the hypotheses of Theorem 2.

Proof. Suppose there is such a decomposition; we derive a contradiction. Recall that $B$ is an orientable $k$-gm. Now apply the Leray spectral sequence with the families $\phi, \psi$ of compact supports on $B, M$. In particular, there is an injection

$$
\alpha: H_{c}^{0}\left(B ; \mathscr{\ell}^{k-1}\right) \rightarrow H_{c}^{k}(B) .
$$

The cokernel of $\alpha$ is imbedded in $H_{c}^{k}\left(E^{n+k}\right)=0$; thus, $\alpha$ is an isomorphism. Obviously, $H_{c}^{0}\left(B ; \mathscr{H}^{k-1}\right) \cong 0$, since $\mathscr{H}^{k-1}$ is Hausdorff and $B$ is noncompact. By duality, $H_{c}^{k}(B) \cong H_{0}(B) \cong \mathbf{Z}$. This is a contradiction.

We now give some examples which are not wholly satisfying.

Example 3.4.5. Let $X$ be a $k$-gm $(k \geq 3)$ so that $X \times E^{k-1}$ is a manifold. Fix $j>0$; for each $1 \leq i \leq j$, let $\Sigma_{i}^{d_{i}}$ be a homology $\left(d_{i}\right)$-sphere, where $d_{i} \geq k-1$. Let $n=d_{1}+d_{2}+\cdots+d_{j}$. Let

$$
M=X \times\left(\prod_{i=1}^{j} \Sigma_{i}\right)
$$

then $M$ is an $(n+k)$-manifold. Let $G$ be the following product decomposition:

$$
G=\left\{x \times\left(\prod_{i=1}^{j} \Sigma_{i}\right) \mid x \in X\right\}
$$

The decomposition space is obviously $X$.

Example 3.4.6. Let $G_{0}$ be any uscd of $N^{2 k-1}$ into homology $(k-1)$-spheres $(k \geq 3)$; see $\S 2.4$ for explicit examples. Let

$$
M^{n+k}=N^{2 k-1} \times \Sigma^{n-k+1},
$$

where $\Sigma$ is a homology $(n-k+1)$-sphere and $n>2 k-2 \geq 4$. Let

$$
G=\left\{g \times \Sigma^{n-k+1} \mid g \in G_{0}\right\} ;
$$

then $G$ is a uscd of $M$ into homology $\left(S^{k-1} \times S^{n-k+1}\right)$ 's. Obviously, $M / G \cong$ $N^{2 k-1} / G_{0}$, a $k$-gm.

Questions. (1) Does Theorem 2 remain valid if the hypothesis concerning nondegeneracy is dropped? (Perhaps if we assume $B$ to be an ANR.) 
(2) Is there an approximate fibration defined on $S^{n+k}$ with fiber the shape of $S^{k-1} \times S^{n-k+1}$ ? More generally, is there a decomposition of $S^{n+k}$, in addition to satisfying the hypotheses of Theorem 2, such that $\operatorname{rank} H_{k-1}(g)=1$ for some or all $g \in G$ ? (This is really the case of interest in light of 3.4 .1 and 3.4.3.)

(3) If $G$ were a uscd of $S^{n+k}$ satisfying the hypotheses of Theorem 2, except that $K \neq \varnothing$, then can $K$ contain more than one point? (I.e., is there an analogue of 2.4.2?)

(4) Under what conditions is $\left.p\right|_{M-\widetilde{E}}$ an approximate fibration? In particular, if each decomposition element has the shape of a simply-connected closed $n$ manifold and $M=S^{n+k}$, is $\left.p\right|_{M-\widetilde{E}}$ an approximate fibration?

\section{BIBLIOGRAPHY}

[A] F. Ancel, The locally flat approximation of cell-like embedding relations, Ph.D. thesis, University of Wisconsin, Madison, 1976.

[BoSb] F. Bonahon and L. Siebenmann, The classification of Seifert fibred 3-orbifolds, Low Dimensional Topology (R. A. Fenn, ed.), London Math. Soc. Lecture Note Ser., vol. 95, Cambrige Univ. Press, Cambridge, 1985, pp. 19-85.

[Br1] G. Bredon, Sheaf theory, McGraw-Hill, New York, 1967.

[Br2] - Generalized manifolds revisited, Topology of Manifolds (Proc. Univ. of Georgia Topology of Manifolds Inst., 1969) (J. C. Cantrell and C. H. Edwards, eds.), Markham, Chicago, Ill., 1970, pp. 461-469.

[C] J. W. Cannon, $\Sigma^{2} H^{3}=S^{5} / G$, Rocky Mountain J. Math. 8 (1978), 527-532.

[CD1] D. S. Coram and P. F. Duvall, Approximate fibrations, Rocky Mountain J. Math. 7 (1977), 275-288.

[CD2] _ Non-degenerate $k$-sphere mappings, Topology Proc. 4 (1979), 67-82.

[CD3] Mappings from $S^{3}$ to $S^{2}$ whose point inverses have the shape of a circle, General Topology Appl. 10 (1979), 239-246.

[CD4] _ Finiteness theorems for approximate fibrations, Trans. Amer. Math. Soc. 269 (1982), 383-394.

[D1] R. J. Daverman, Decompositions of manifolds into codimension one manifolds, Compositio Math. 55 (1985), 185-207.

[D2] The three dimensionality of certain codimension three decompositions, Proc. Amer. Math. Soc. 96 (1986), 175-179.

[D3] _ Decompositions of manifolds, Academic Press, Orlando, Fla., 1986.

[D4] _ Decompositions into submanifolds of fuxed codimension, Geometric and Algebraic Topology, Banach Center Publications, no. 18, PWN-Polish Scientific Publishers, Warsaw, 1986, pp. 109-116.

[DH] R. J. Daverman and L. S. Husch, Decompositions and approximate fibrations, Michigan Math. J. 31 (1984), 197-214.

[DW1] R. J. Daverman and J. J. Walsh, A ghastly generalized n-manifold, Illinois J. Math. 25 (1981), 555-576.

[DW2] _ Decompositions into codimension two spheres and approximate fibrations, Topology Appl. 19 (1985), 103-121.

[DW3] , Decompositions of manifolds into codimension-2 submanifolds, Trans. Amer. Math. Soc. 288 (1985), 273-291.

[DW4] , Manifold decompositions yielding generalized manifolds, Topology Appl. 26 (1987), 143-162. 
[Dy] J. Dydak, On LC $C^{n}$-divisors, Topology Proc. 3 (1978), 319-333.

[DyS1] J. Dydak and J. Segal, Shape theory: An introduction, Lecture Notes in Math., vol. 688, Springer-Verlag, Berlin, 1978.

[DyS2] __ Local n-connectivity of decomposition spaces, Topology Appl. 18 (1984), 43-58.

[DyW] J. Dydak and J. J. Walsh, Sheaves that are locally constant with applications to homology manifolds, Geometric Topology and Shape Theory (S. Mardešić and J. Segal, eds.), Lecture Notes in Math., vol. 1283, Springer-Verlag, Berlin, 1987, pp. 65-87.

[E] R. D. Edwards, Suspensions of homology spheres, unpublished manuscript.

[Gr] M. J. Greenberg and J. R. Harper, Algebraic topology, Benjamin/Cummings, Menlo Park, Calif., 1981.

[Ho] H. Hopf, Über die Abbildungen der 3-dimensionalen Sphäre auf die Kugelfläche, Math. Ann. 104 (1931), 637-665.

[Hu] Sze-Tsen Hu, Theory of retracts, Wayne State Univ. Press, Detroit, Mich., 1965.

[H] T. W. Hungerford, Algebra, Springer-Verlag, New York, 1974.

[Hr] W. Hurewicz, Homotopie, Homologie, und lokaler Zussamenhang, Fund. Math. 25 (1935), 467-485.

[Hus] D. Husemoller, Fibre bundles, 2nd ed., Springer-Verlag, New York, 1975.

[L1] R. C. Lacher, $k$-sphere mappings on $S^{2 k+1}$, (Proc. Utah Geom. Topology. Conf., 1974) (L. C. Glaser and T. B. Rushing, eds.), Lecture Notes in Math., vol. 438, Springer-Verlag, Berlin, 1974.

[McC] John McCleary, User's guide to spectral sequences, Publish or Perish, Wilmington, Del., 1985.

[N] Keio Nagami, Dimension theory, Academic Press, New York, 1970.

[Na] Jun-Iti Nagata, Modern dimension theory, revised and extended ed., Heldermann-Verlag, Berlin, 1983.

[R] J. J. Rotman, Introduction to homological algebra, Academic Press, New York, 1979.

[Sc] G. P. Scott, The geometries of 3-manifolds, Bull. London Math. Soc. 15 (1983), 401-487.

[Se] H. Seifert, Topologie dreidimensionaler gefaserter Räume, Acta Math. 60 (1932), 147-238.

[S] E. H. Spanier, Algebraic topology, McGraw-Hill, 1966.

[Sw] R. Swan, Sheaf theory, Univ. of Chicago Press, Chicago, Ill., 1964.

[Th] William P. Thurston, The geometry and topology of three-manifolds, lecture notes.

[W] R. L. Wilder, Topology of manifolds, rev. ed., 4th printing, Amer. Math. Soc., Providence, R. I., 1949.

Department of Mathematics, Southwest Texas State University, San Marcos, Texas 